\title{
Orbit design for future SpaceChip swarm missions in a planetary atmosphere ${ }^{1}$
}

\author{
Camilla Colombo $^{2}$, Colin McInnes ${ }^{3}$ \\ Advanced Space Concepts Laboratory, University of Strathclyde, Glasgow, United Kingdom
}

\begin{abstract}
The effect of solar radiation pressure and atmospheric drag on the orbital dynamics of satellites-on-a-chip (SpaceChips) is exploited to design equatorial long-lived orbits about the oblate Earth. The orbit energy gain due to asymmetric solar radiation pressure, considering the Earth's shadow, is used to balance the energy loss due to atmospheric drag. Future missions for a swarm of SpaceChips are proposed, where a number of small devices are released from a conventional spacecraft to perform spatially distributed measurements of the conditions in the ionosphere and exosphere. It is shown that the orbit lifetime can be extended and indeed selected through solar radiation pressure and the end-of-life re-entry of the swarm can be ensured, by exploiting atmospheric drag.
\end{abstract}

Keywords: solar radiation pressure; atmospheric drag; $J_{2}$; Earth's oblateness; equilibrium orbit; swarm; MEMS; SpaceChip.

\section{Introduction}

Recent innovations in spacecraft design exploit advances in miniaturisation to fabricate small satellites with dimensions of a single chip [1]. In addition, current concepts for MEMS (micro-electromechanical systems) devices have been designed with micro-power sources, sensing, computing and bi-directional communication capabilities for terrestrial $1 \mathrm{~mm}^{3}$ 'smart dust' applications [2,3]. These existing capabilities have also been exploited to design nearterm concepts for functional devices in space, such as satellites-on-a-chip (SpaceChips) [4-7].

This new technology offers the benefit of low-cost manufacturing of vast number of micro-spacecraft for use in swarm applications. The considerably smaller dimensions of satellites-on-a-chip allow their deployment in orbit from a CubeSat or as piggy-back on a conventional spacecraft, thus allowing significant launch cost savings. Increased launch opportunities and low manufacturing costs reduce total mission cost and hence the employment of 'smart dust' devices can accept higher risk to investigate new phenomena or explore the harsh space environment. The deployment of vast numbers of SpaceChips will enable future missions, such as global sensor networks for Earth observation and communication, distributed space missions for multi-point, real-time sensing for space science (space weather, geomagnetic physics, reflectometry), interplanetary exploration in support of traditional spacecraft, or deployment in the vicinity of a spacecraft for diagnostic or environmental detection purposes.

As an early example of a SpaceChip-scale swarm, project West Ford in 1963 placed a ring of $4.8 \times 10^{8}$ copper dipole antennas $(1.78 \mathrm{~cm}$ long needles, with a diameter of $17.8 \mu \mathrm{m})$ into orbit to allow global radio communication [8]. The motion of the individual dipoles, from dispensing to final re-entry through the atmosphere was both modelled and observed. More recently, Petschek et al. [9] proposed a Kilo-Satellite constellation mission (less than one kilogram satellites) for the distributed measurement of the global instantaneous structure of the magnetosphere.

The realisation of these swarm concepts requires an understanding of orbital dynamics at extremely small spacecraft length-scales. The significantly higher area-to-mass ratio of future SpaceChip or smaller 'smart dust' devices, with respect to conventional spacecraft, requires new insights into orbital dynamics, as perturbations such as solar radiation pressure (SRP) and aerodynamic drag cannot be neglected. On the contrary, length scaling can be exploited to achieve new mission opportunities [10].

The effect of natural perturbations on small particles has been studied extensively in the vast literature on the dynamics of cosmic dust in the solar system whose motion is influenced by solar gravity, solar radiation pressure, the Poynting-Robertson drag force, planetary oblateness and electromagnetic forces [11-13]. The effect of solar

\footnotetext{
${ }^{1}$ A previous version of this paper was presented during the $61^{\text {st }}$ International Astronautical Congress in Prague IAC10.C1.8.2

${ }^{2}$ Corresponding author. Research Fellow. E-mail address: camilla.colombo@strath.ac.uk, Tel. +44 1415485727.

${ }^{3}$ Professor. E-mail address: colin.mcinnes@strath.ac.uk, Tel. +44 1415482049.
} 
radiation pressure, zonal and tesseral harmonics of the Earth's gravitational potential, luni-solar third body perturbations and atmospheric drag on high area-to-mass ratio objects are also of particular interest to explain the long-term dynamical evolution of high area-to-mass debris particles in Earth orbit [14, 15]. Furthermore, the effects of solar radiation pressure have been observed since 1960 in the orbital behaviour of balloon satellites [16-18] and are exploited as the main propulsive force for solar sailing [19-21].

In a previous paper, the orbital dynamics of such 'smart dust' devices was analysed under the effect of SRP and aerodynamic drag in a spherically symmetric gravitational field and the energy input from asymmetric solar radiation pressure was used to offset the energy dissipation of atmospheric drag, without the use of active control [22]. Families of long-lived orbits for swarms of SpaceChips were presented for which the condition of Sun-synchronous apse-line precession is achieved passively without any propellant mass consumption. A comparison with the dragonly and SRP-only scenarios showed that the exploitation of the natural effects of solar radiation pressure and atmospheric drag provides a means of enabling long-lived orbits for future SpaceChip and 'smart dust' devices without the use of active control, and ensures the final re-entry of the devices so that they do not constitute future space debris. Due to the large area-to-mass ratio of these devices orbit lifetime due to air drag alone is extremely short.

Using these findings, in this paper the natural effects of solar radiation pressure and atmospheric drag perturbations on high area-to-mass objects are exploited to design swarm missions for the mapping and study of the upper regions of the Earth's atmosphere. A swarm of SpaceChips is proposed as distributed nodes of a sensor network to obtain a spatial and temporal map of the ionosphere and exosphere. The model adopted in [22] is here improved and extended to consider also the influence of the Earth's oblateness (i.e., the second zonal harmonic coefficient $J_{2}$ ). Therefore, a new family of equilibrium orbits can be identified at low altitudes, where the influence of planetary oblateness is significant [11-13, 18]. Given the initial orbital elements of the spacecraft, the shadow geometry is given as a function of semi-major axis, eccentricity and angular displacement between the Sun-Earth line and the orbit pericentre. The secular change of the in-plane orbital elements over a single orbit revolution is evaluated analytically in the presence of Earth's shadow. The coupled effect of atmospheric drag and solar radiation pressure, with asymmetry due to eclipses, in an oblate gravity field is exploited to disperse the 'smart dust' devices in the Earth's atmosphere, to greatly extend the orbit lifetime of such devices and to assess and design the disposal of the devices at the end of the mission. Importantly, the short lifetime of high area-to-mass spacecraft can be greatly extended and indeed selected through the interaction of energy gain from asymmetric solar radiation pressure and energy dissipation due to drag.

The paper is organised as follows: Section 2 introduces the analytical approach to compute the long-term orbit evolution under the effect of SRP, $J_{2}$ and atmospheric drag; Section 3 presents the conditions for equilibrium orbits and gives some insights in the long-term orbit evolution of set of initial conditions in the element space. This section presents also a discussion on how the effect of the Earth's oblateness influences previously presented results which consider only solar radiation pressure and drag [22]. In Section 4 the effect of natural perturbations is exploited to design swarm missions for the study of the upper regions of the atmosphere, thus offering a deeper representation of element space analysis performed in the first part of the paper.

\section{Orbital dynamics}

The orbit evolution of the spacecraft is obtained through an averaging technique with Gauss' planetary equations to obtain the secular variation of the orbital elements. In a previous paper, as a first approach to explore orbital dynamics at extremely small length-scales, only solar radiation pressure and the atmospheric drag were considered [22]. These perturbing accelerations are proportional to the area-to-mass ratio of the spacecraft; hence their effect is greater for small devices. The model considered here includes also the influence of the Earth's oblateness represented by the second zonal harmonic coefficient $J_{2}$, which acts as an important perturbation at perigee altitudes lower than approximately three Earth radii [23]. To begin to explore conditions for long-lived orbits we constrain the present study to a planar problem. In particular we consider orbits with zero inclination and the equatorial plane is assumed to be in the ecliptic (i.e., the obliquity angle of the ecliptic with respect to the equator is set to zero). This latter approximation holds when the obliquity angle is less than about 30 degrees [23, 24]. The first step is to define the secular rate of change in orbital elements due to solar radiation pressure, drag and Earth's oblateness; this will be addressed in the remainder of this section. For completeness, we report here the model of solar radiation pressure and atmospheric drag considered, already presented in [22]; we then introduce the equations of the secular rate of change of Keplerian elements due to $J_{2}$. 


\subsection{Solar radiation pressure}

We consider a spacecraft on an Earth-centred orbit lying in the equatorial plane (which is approximated to be on the ecliptic plane) as represented in Fig. 1. The satellite is subjected to the acceleration due to solar radiation pressure given by

$$
\begin{aligned}
& a_{r, \mathrm{SRP}}=a_{\mathrm{SRP}} \cos (\phi+f) \\
& a_{\theta, \mathrm{SRP}}=-a_{\mathrm{SRP}} \sin (\phi+f)
\end{aligned}
$$

where $f$ is the true anomaly, $a_{r}$ and $a_{\theta}$ are the components of the acceleration along the radial and transverse direction in the orbital plane, and $a_{\mathrm{SRP}}$ is the characteristic acceleration

$$
a_{\mathrm{SRP}}=\frac{p_{\mathrm{SR}} c_{R} A_{\odot}}{m}
$$

where $p_{\mathrm{SR}}=4.56 \times 10^{-6} \mathrm{~N} / \mathrm{m}^{2}$ is the solar pressure at $1 \mathrm{AU}, c_{\mathrm{R}}$ the reflectivity coefficient, $A_{\odot}$ is the area exposed to the Sun, and $m$ is the mass of the satellite. The angle $\phi=\tilde{\omega}-\lambda_{\text {Sun }}$ represents the angular displacement between the orbit pericentre (being a planar problem we can define the longitude of the pericentre $\tilde{\omega}$ as shown in Fig. 1) and the Sun-Earth direction $\lambda_{\text {Sun }}$.

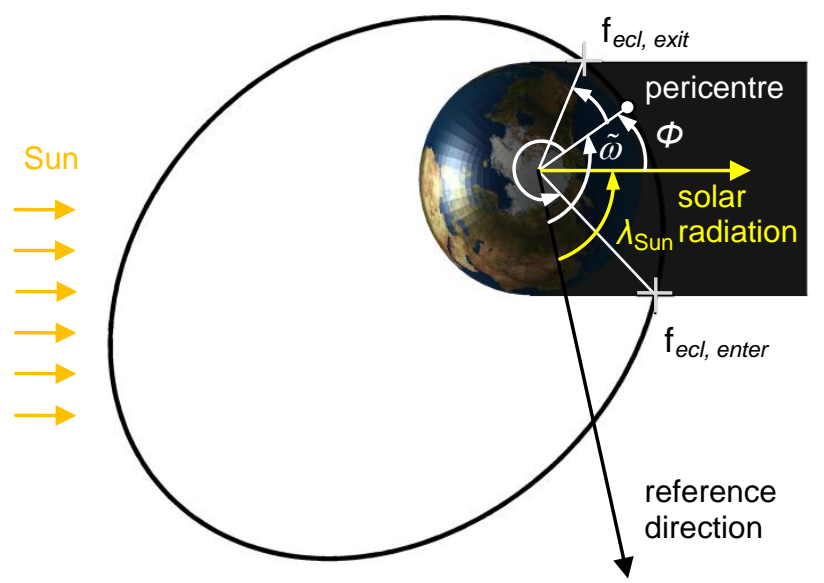

Fig. 1 Orbit geometry.

The effect of SRP on the spacecraft orbit is expressed in terms of the long-term variation of the orbital elements. Gauss' equations for the variation of semi-major axis $a$, eccentricity $e$ and longitude of the pericentre are given by [25]

$$
\begin{aligned}
& \frac{d a}{d f}=\frac{2 p r^{2}}{\mu_{\text {Earth }}\left(1-e^{2}\right)^{2}}\left(e \sin f \cdot a_{r}+\frac{p}{r} \cdot a_{\theta}\right) \\
& \frac{d e}{d f}=\frac{r^{2}}{\mu_{\text {Earth }}}\left(\sin f \cdot a_{r}+\left(1+\frac{r}{p}\right) \cos f \cdot a_{\theta}+e \frac{r}{p} \cdot a_{\theta}\right) \\
& \frac{d \tilde{\omega}}{d f}=\frac{r^{2}}{\mu_{\text {Earth }} e}\left(-\cos f \cdot a_{r}+\left(1+\frac{r}{p}\right) \sin f \cdot a_{\theta}\right)
\end{aligned}
$$

with $\mu_{\text {Earth }}$ the gravitational parameter of the Earth, $r$ the orbit radius, $p$ the semilatus rectum and the acceleration components given by Eq. (1). Eqs. (3) are integrated in average over true anomaly $f$ considering the other orbital elements to be constant over one orbit revolution. In the case of a constant disturbing acceleration in a fixed inertial direction, Gauss' equations can be solved in closed form to find the primitive functions fun $_{a}$, fun ${ }_{e}$, fun $\tilde{\omega}_{\tilde{\omega}}$ for semimajor axis, eccentricity and longitude of the pericentre: 


$$
\begin{aligned}
& \operatorname{fun}_{a}(a, e, \phi)=\int \frac{d a}{d f} d f+c_{a} \\
& \operatorname{fun}_{e}(a, e, \phi)=\int \frac{d e}{d f} d f+c_{e} \\
& \operatorname{fun}_{\tilde{\omega}}(a, e, \phi)=\int \frac{d \tilde{\omega}}{d f} d f+c_{\tilde{\omega}}
\end{aligned}
$$

Some integration constants $c_{a}, c_{e}$ and $c_{\tilde{\omega}}$ are introduced in Eqs. (4) but are then removed when the primitive function is evaluated at the two limits of integration. The indefinite integrals in Eqs. (4) were computed in [22] as:

$$
\begin{aligned}
\text { fun }_{a}= & -\frac{2 a^{3}\left(1-e^{2}\right)}{\mu_{\text {Earth }}} a_{\mathrm{SRP}} \frac{1}{e(1+e \cos f)}(\cos \phi+e \sin \phi \sin f)+c_{a} \\
\text { fun }_{e}= & \frac{a^{2}\left(1-e^{2}\right)^{2}}{\mu_{\text {Earth }}} a_{\mathrm{SRP}}\left(\sin \phi\left(-\frac{3}{2} \frac{E}{\left(1-e^{2}\right)^{3 / 2}}+\frac{\sin f\left(\cos f\left(-8 e^{4}+10 e^{2}-2\right)+6 e\left(1-e^{2}\right)\right)}{4\left(1-e^{2}\right)^{2}(1+e \cos f)^{2}}\right)\right. \\
& \left.-\frac{\cos \phi}{2 e^{2}(1+e \cos f)^{2}}\left(1+2 e \cos f+e^{2}\right)\right)+c_{e} \\
\text { fun }_{\tilde{\omega}}=- & \frac{a^{2}\left(1-e^{2}\right)^{2}}{\mu_{\text {Earth }} e} a_{\mathrm{SRP}}\left(\cos \phi\left(\frac{3}{2} \frac{E}{\left(1-e^{2}\right)^{3 / 2}}-\frac{e \sin f}{\left(1-e^{2}\right)(1+e \cos f)}-\frac{1}{2} \frac{(e+\cos f) \sin f}{\left(1-e^{2}\right)(1+e \cos f)^{2}}\right)\right. \\
& \left.+\sin \phi \frac{1+2 e \cos f}{2 e^{2}(1+e \cos f)^{2}}\right)+c_{\tilde{\omega}}
\end{aligned}
$$

where $E$ is the eccentric anomaly which is a function of the true anomaly. Note that Eqs. (5) have been obtained under the assumption that the orbit lies in the ecliptic plane. Eqs. (5) assumes that the disturbing acceleration $a_{\mathrm{SRP}}$ is constant when the spacecraft is in sunlight, i.e., the variation of the solar flux over time is neglected, and the exposed area $A_{\odot}$ in Eq. (2) is considered constant (i.e., the spacecraft has a spherical shape or the attitude of the spacecraft is constant with respect to the Sun-Earth line). The total variation of the orbital elements can be evaluated over the orbit arc in which the spacecraft is in sunlight: $\left[0, f_{\text {ecl, enter }}\right],\left[f_{\text {ecl, exit }}, 2 \pi\right]$, where $f_{\text {ecl, enter }}$ and $f_{\text {ecl, exit }}$ are the true anomalies at which the satellite enters and exits the eclipses. Since we are assuming the orbital elements and $a_{\mathrm{SRP}}$ to be fixed over one orbit revolution at their value at the pericentre (i.e., averaging technique), the total variation of the orbital elements can be written as:

$$
\begin{aligned}
& \Delta a_{\mathrm{SRP}, 2 \pi}\left(a, e, \phi, f_{\text {ecl, exit }}, f_{\text {ecl, enter }}\right)=\left[\mathrm{fun}_{a}\right]_{f_{\text {ccl, exit }}}^{f_{\text {ece ener }}} \\
& \Delta e_{\mathrm{SRP}, 2 \pi}\left(a, e, \phi, f_{\text {ecl, exit }}, f_{\text {ecl, enter }}\right)=\left[\text { fun }_{e}\right]_{f_{\text {ecl, exit }}}^{f_{\text {cel enter }}} \\
& \Delta \tilde{\omega}_{\mathrm{SRP}, 2 \pi}\left(a, e, \phi, f_{\text {ecl, exit }}, f_{\text {ecl, enter }}\right)=\left[\mathrm{fun}_{\tilde{\omega}}\right]_{f_{\text {ecl, exit }}}^{f_{\text {fel ener }}}
\end{aligned}
$$

In the planar case we consider, assuming that the parallax of the Sun is negligible, the arguments of true anomaly at which the satellite enters and exits the Earth's shadow are given by the following conditions (see Fig. 1):

$$
\begin{aligned}
& f_{\text {ecl, exit }}(a, e, \phi):\left\{\begin{array}{l}
r \cos (\phi+f)>0 \\
r \sin (\phi+f)=+R_{\text {Earth }}
\end{array}\right. \\
& f_{\text {ecl, enter }}(a, e, \phi):\left\{\begin{array}{l}
r \cos (\phi+f)>0 \\
r \sin (\phi+f)=-R_{\text {Earth }}
\end{array}\right.
\end{aligned}
$$


where $R_{\text {Earth }}$ is the mean radius of the Earth, and $r$ the orbit radius. The expressions for $f_{\text {ecl, exit }}(a, e, \phi)$ and $f_{\text {ecl, enter }}(a, e, \phi)$ can be expressed as a closed-form function of the orbital elements $a, e$, and $\phi$ as given in [22] and can be substituted into Eqs. (6) to give the actual variation of orbital elements considering the Earth's shadow as function of $a, e$, and $\phi$ :

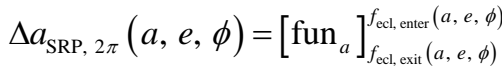

$$
\begin{aligned}
& \Delta e_{\mathrm{SRP}, 2 \pi}(a, e, \phi)=\left[\mathrm{fun}_{e}\right]_{f_{\text {cel, exit }}(a, e, \phi)}^{f_{\mathrm{cl}, \text { nere }}(a, e, \phi)} \\
& \Delta \tilde{\omega}_{\mathrm{SRP}, 2 \pi}(a, e, \phi)=\left[\mathrm{fun}_{\tilde{\omega}}\right]_{f_{\text {ccl, exit }}(a, e, \phi)}^{f_{\text {cle enter }}(a, e, \phi)}
\end{aligned}
$$

If the spacecraft does not enter into eclipse (i.e., $f_{\text {ecl, enter }}=f_{\text {ecl, exit }}+2 \pi$ ) some terms of Eqs. (8) vanish; in particular the variation of semi-major axis is zero.

\subsection{Atmospheric drag}

For spacecraft orbits with a low perigee the motion is also influenced by atmospheric drag acceleration

$$
\mathbf{a}_{\text {Drag }}=-\frac{1}{2} \frac{c_{D} A_{\text {Drag }}}{m} \rho v_{r e l}^{2} \hat{\mathbf{v}}_{\text {rel }}
$$

where $c_{D}$ is the drag coefficient, $A_{\text {Drag }}$ is the effective cross-sectional area of the spacecraft and $m$ its mass, $v_{r e l}$ is the velocity relative to the rotating atmosphere and $\hat{\mathbf{v}}_{\text {rel }}$ the corresponding unit vector. The secular disturbing effect on the orbit due to atmospheric drag can be modelled analogously to the case of solar radiation pressure. Starting from Gauss' equations written as function of the eccentric anomaly, King-Hele [26] derived equations which express the secular perturbation on the orbital elements due to atmospheric drag. These equations are based on the assumption of a time-independent, spherically-symmetric atmosphere with a density that varies exponentially with altitude $h$, according to:

$$
\rho=\rho_{0} \exp \left[-\frac{h-h_{0}}{H}\right]
$$

where $\rho_{0}$ is the reference density at the reference altitude $h_{0}$ and $H$ is the scale height, whose values are taken from tables [27]. If we neglect atmospheric rotation (i.e., the angular velocity of the Earth is zero), the variation of $\Delta \tilde{\omega}_{\text {Drag, } 2 \pi}$ due to drag is zero, while the change of the in-plane orbital elements over a single revolution is given by [28]:

$$
\begin{aligned}
\Delta e_{\text {Drag, } 2 \pi} & =-2 \pi \delta a \rho_{p} \exp [-c]\left\{I_{1}+\frac{e}{2}\left(I_{0}+I_{2}\right)-\frac{e^{2}}{8}\left(5 I_{1}-I_{3}\right)-\frac{e^{3}}{16}\left(5 I_{0}+4 I_{2}-I_{4}\right)+O\left(e^{4}\right)\right\} \\
\Delta h_{p \text { Drag, } 2 \pi} & =-2 \pi \delta a^{2} \rho_{p} \exp [-c]\left\{\left(I_{0}-I_{1}\right)-\frac{e}{2}\left(3 I_{0}-4 I_{1}+I_{2}\right)+\frac{e^{2}}{8}\left(6 I_{0}-11 I_{1}+6 I_{2}-I_{3}\right)+\right. \\
& \left.-\frac{e^{3}}{16}\left(7 I_{0}-12 I_{1}+8 I_{2}-4 I_{3}+I_{4}\right)+O\left(e^{4}\right)\right\} \\
\Delta a_{\text {Drag, } 2 \pi} & =\frac{\Delta h_{p \text { Drag, } 2 \pi}+a \cdot \Delta e_{\text {Drag, } 2 \pi}}{1-e} \\
\Delta \tilde{\omega}_{\text {Drag, } 2 \pi} & =0
\end{aligned}
$$

where $h_{p}$ is the altitude of perigee, $\rho_{p}$ is the density at the orbit perigee, computed through Eq. (10), the factor $c=a e / H, I_{k}$ are the modified Bessel functions of the first kind of order $k$ and argument $c$ [29], and $\delta=Q A_{\text {drag }} c_{D} / m$ (where the drag coefficient $c_{D}$ is considered constant, and the factor $Q$ is equal to 1 for a static 
atmosphere). Eqs. (11) are valid up to the order of eccentricity indicated, within the range $0.01 \leq e \leq 0.8$. Note that the assumption of vanishing angular rate of the Earth could be removed since the orbit considered here is equatorial and we are neglecting the obliquity angle of the ecliptic and the equator. Without this latter assumption, the out-ofplane components of a rotating atmosphere with respect to an inclined orbit should be considered.

\subsection{Earth's oblateness}

Since orbits in the equatorial plane are considered (zero inclination), the effect of orbital regression and rotation of perigee caused by the Earth's oblateness can be added up to give the change in longitude of the pericentre $\tilde{\omega}$ that is measured on the equatorial plane with respect to a fixed inertial direction:

$$
\begin{aligned}
& \Delta e_{J_{2}, 2 \pi}=0 \\
& \Delta a_{J_{2}, 2 \pi}=0 \\
& \Delta \tilde{\omega}_{J_{2}, 2 \pi}=\frac{3 R_{\text {Earth }}^{2} J_{2} \pi}{a^{2}\left(1-e^{2}\right)^{2}}
\end{aligned}
$$

where $J_{2}=1.083 \times 10^{-3}$ denotes the second zonal harmonic coefficient and $R_{\text {Earth }}$ is the mean radius of the Earth. It is interesting to note that in the equatorial plane the rate of change of the argument of the ascending node due to $J_{2}$ is negative, equal to one half of the rate of change of the anomaly of perigee [30].

\subsection{Secular variation of the orbital elements}

The total variation of the orbital elements over a single orbit revolution can be computed by adding Eqs. (8), (11) and (12), under the assumption, commonly adopted in averaging techniques, that the coupling between different perturbations is negligible to first order [27]. Then the secular and long-period rate of change of the orbital elements can be obtained by dividing by the Keplerian orbital period

$$
T=2 \pi \sqrt{\frac{a^{3}}{\mu_{\text {Earth }}}}
$$

thus giving

$$
\begin{aligned}
& \left\langle\frac{d a}{d t}\right\rangle_{\mathrm{SRP}+\text { Drag }}= \\
& \left\langle\frac{d e}{d t}\right\rangle_{\mathrm{SRP}+\text { Drag }}=\frac{\Delta a_{\mathrm{SRP}, 2 \pi}+\Delta a_{\mathrm{Drag}, 2 \pi}}{2 \pi} \sqrt{\frac{\mu_{\text {Earth }}}{a^{3}}} \\
& \left\langle\frac{d \tilde{\omega}}{d t}\right\rangle_{\mathrm{SRP}+J_{2}}=\frac{\Delta \tilde{\omega}_{\mathrm{SRP}, 2 \pi}+\Delta \tilde{\omega}_{J_{2}, 2 \pi}}{2 \pi} \sqrt{\frac{\mu_{\text {Earag }, 2 \pi}}{a^{3}}}
\end{aligned}
$$

where the angular brackets denote the secular variation. The validity of Eqs. (14) was verified by comparison with numerical integration of the dynamics in Cartesian coordinates, using the expression for the disturbing accelerations Eq. (1), Eq. (9) (see [31]), and Eq. (12). The line of apsides of the ellipse will rotate due to the perturbing solar radiation pressure and $J_{2}$ acceleration, with a mean rate of precession given by the Eq. (14c) that can be rewritten to express the perigee precession with respect to the Sun-Earth line, introducing the orbital rate of the Earth around the Sun $n_{\text {Sun }}$ :

$$
\left\langle\frac{d \phi}{d t}\right\rangle_{\mathrm{SRP}+J_{2}}=\left\langle\frac{d \tilde{\omega}}{d t}\right\rangle_{\mathrm{SRP}+J_{2}}-n_{\mathrm{Sun}}
$$




\section{Long-lived orbits under the effect of solar radiation pressure, $J_{2}$, and drag}

To analyse the influence on the satellite's orbit of asymmetric solar radiation pressure and atmospheric drag in an oblate gravity field, it is convenient to first identify the equilibrium conditions of the associated system Eq. (14). Those solutions represent equilibrium orbits under the effect of these perturbations. Afterwards, through the study of the derivative sign and numerical integration of the secular variation of orbital elements, it will be possible to characterise the long-term evolution of other initial conditions (different to the equilibrium orbits) in the space of the orbital elements.

The equilibrium conditions for 'smart dust' devices with solar radiation pressure and atmospheric drag, together with a characterisation of families of long-lived orbits, were analysed in [22]. In the following section we will adopt the same method to investigate how the effect of the Earth's oblateness affects those equilibrium solutions.

\subsection{Conditions for equilibrium orbits}

The search for equilibrium orbits imposes three conditions to be satisfied. The total variation of semi-major axis and eccentricity due to SPR and drag must be zero, i.e., the combined effect of the two natural perturbations must cancel. Moreover, the orbit must keep the same orientation relative to the Sun during a year, i.e., the secular rate of longitude of the perigee due to solar radiation pressure and $J_{2}$ (recall from Eqs. (11) that $\Delta \tilde{\omega}_{\text {Drag, } 2 \pi}=0$ ), must be equal to the orbital rate of the Earth around the Sun (i.e., the apparent motion of the Sun around an Earth-centred inertial system), such that the net change in $\phi$ is zero (i.e., Sun-synchronous apse-line precession). If this would not be the case, the orbit apse-line would drift with respect to the Sun-Earth direction. As a consequence, the secular perturbation due to SRP would change (as it depends on $\phi$ ) and, since the perturbation of $J_{2}$ and drag is independent of $\phi$, the orbit would no longer be in equilibrium. Note that the condition of Sun-synchronous apse-line precession required here is different to the condition of Sun-synchronicity which requires instead that only the change in anomaly of the ascending node equals the rate of rotation of the Earth around the Sun. The condition of Sunsynchronous apse-line precession guarantees that the line of apsides is at a constant orientation with respect to the Sun.

$$
\left\{\begin{array}{l}
\left\langle\frac{d a}{d t}\right\rangle_{\mathrm{SRP}+\mathrm{Drag}}=0 \\
\left\langle\frac{d e}{d t}\right\rangle_{\mathrm{SRP}+\mathrm{Drag}}=0 \\
\left\langle\frac{d \tilde{\omega}}{d t}\right\rangle_{\mathrm{SRP}+J_{2}}-n_{\mathrm{Sun}}=0
\end{array}\right.
$$

Note that Eq. (16c) in the simplified case without Earth shadow and neglecting $J_{2}$ was adopted by McInnes et al. [19] and Oyama et al. [20] for determining the required characteristic acceleration of a solar sail to provide Sunsynchronous apse-line precession.

An analysis of the change in orbital elements due to solar radiation pressure and atmospheric drag as a function of the initial conditions of the satellite (i.e., the values of the Keplerian elements $a, e$, and $\phi$ ) was performed in [22]. From the study of the sign of $\Delta a_{\mathrm{SRP}, 2 \pi}, \Delta a_{\mathrm{Drag}, 2 \pi}, \Delta e_{\mathrm{SRP}, 2 \pi}, \Delta e_{\mathrm{Drag}, 2 \pi}$, it was possible to define the domain of angular displacements $\phi$ where the first two equations of system Eq. (16) can be satisfied.

- The total change in semi-major axis Eq. (16a) can be zero within the range $0 \leq \phi \leq \pi$.

- The total variation of eccentricity Eq. (16b) can be zero for $\pi \leq \phi \leq 2 \pi$.

In both cases, the equality conditions $\phi=0,2 \pi$ and $\phi=\pi$, are satisfied corresponding to perigee altitudes where the effect of atmospheric drag can be neglected and only the perturbation of solar radiation pressure has some effect on semi-major axis and eccentricity. Therefore, we can already state that Eq. (16) cannot be satisfied in the region of altitudes where atmospheric drag is present.

The condition of Sun-synchronous apse-line precession Eq. (16c) needs instead a different analysis to that presented in Ref. [22] as the Earth's oblateness is now considered.

The solution of Eqs. (16), which identifies the conditions for equilibrium orbits can be numerically computed through a global multi-start approach. A local algorithm is started from several points randomly distributed over the 
entire domain of semi-major axis, eccentricity and $\phi$. The numerical solution is made possible by the use of the semi-analytical approach explained in Section 2. Alternatively, the use of full numerical integration for determining the change in orbital elements over a single orbit and the numerical solution of the eclipse geometry would make the computational time impractical. Starting from each point on the mesh, a local minimisation is performed numerically $[32,33]$. The objective function used for the minimisation is:

$$
\min _{\{e, \phi, a\}}\left[w_{a}\left|\langle d a / d t\rangle_{\mathrm{SRP}+\mathrm{Drag}}\right|+w_{e}\left|\langle d e / d t\rangle_{\mathrm{SRP}+\mathrm{Drag}}\right|+w_{\phi}\left|\langle d \phi / d t\rangle_{\mathrm{SRP}+J_{2}}\right|\right]
$$

where $\langle d \phi / d t\rangle_{\mathrm{SRP}+J_{2}}$ is given by Eq. (15), and $w_{a}, w_{e}, w_{\phi}$ are weight parameters introduced to treat this multiobjective minimisation problem as a single-objective minimisation (i.e., the three equations of system (16) must be solved).

\subsection{Results for equilibrium and partial equilibrium orbits}

The solution of Eq. (16) can be represented in the space $\{e, \phi, a\}$, or equivalently $\left\{e, \phi, h_{p}\right\}$, as depicted in Fig. 2 , for a spacecraft whose characteristics are reported in Section 4.1. The dotted blue surface on the domain $0 \leq \phi \leq \pi$ represents the solution of $\langle d a / d t\rangle_{\mathrm{SRP}+\mathrm{Drag}}=0$, the dotted grey surface on the domain $\pi \leq \phi \leq 2 \pi$ represents the solutions for which $\langle d e / d t\rangle_{\mathrm{SRP}+\text { Drag }}=0$, and the dotted pink surface is the solution of the Sun-synchronous apse-line precession $\langle d \phi / d t\rangle_{\mathrm{SRP}+J_{2}}{ }^{4}$. While the first two equations of system Eq. (16) are only dependent on SRP and drag, one can note the difference in the solution of the third equation, Eq. (16c), when an oblate gravity field is also considered by comparing Fig. 8 in Ref. [22] to Fig. 2 here. For perigee altitudes higher than approximately three Earth radii, solar radiation pressure is the greatest perturbative effect hence the solution of $\langle d \phi / d t\rangle_{\mathrm{SRP}+J_{2}}$ can be well approximated by the solution of $\langle d \tilde{\omega} / d t\rangle_{\mathrm{SRP}}-n_{\mathrm{Sun}}=0$ where $J_{2}$ is neglected. Within this range of altitudes, the surface $\langle d \phi / d t\rangle_{\mathrm{SRP}+J_{2}}$ extends on the domain $\pi / 2 \leq \phi \leq 3 \pi / 2$. For lower perigee altitudes, instead, the effect of the Earth's oblateness becomes important; the surface $\langle d \phi / d t\rangle_{\mathrm{SRP}+J_{2}}$ extends its domain to the full range of orbit orientations $0 \leq \phi \leq 2 \pi$.

The intersections of the three surfaces in Fig. 2 represent the set of conditions for equilibrium orbits under the effect of asymmetric SRP, drag and $J_{2}$. For high perigee altitudes, a family of solutions of Eq. (16) exists with the condition $\phi=\pi$ (see the intersection of the three surfaces at $\phi=\pi$, represented by the black line). These solutions correspond to orbits with the apse-line along the Sun-Earth direction, and the perigee pointing towards the Sun (antiheliotropic orbits [23]). This position is an equilibrium condition for $\phi$ and the change in eccentricity and semimajor axis over one orbit cancels, as the effect or SRP is symmetric in this configuration, and the drag is negligible; hence the orbit precesses due to SRP alone. At lower perigee altitudes another family of solutions of Eq. (16) exists with the condition $\phi=0$, or equivalently $\phi=2 \pi$ (see the intersection of the three surfaces at $\phi=0,2 \pi$ represented by the black line in Fig. 2). These solutions correspond to orbits with the apse-line along the Sun-Earth direction, and the apogee pointing towards the Sun (heliotropic orbits as the apogee is pointing toward the Sun [23]). In this stable condition the orbit precesses due to SRP and $J_{2}$, while the semi-major axis and the eccentricity remain constant as drag is negligible and the effect of SRP is symmetric in this configuration.

In Fig. 2 the intersection of Eq. (16a) with Eq. (16c) and the intersection of Eq. (16b) with Eq. (16c) are also highlighted (see the black line within the range $0<\phi<\pi$ and the black line within the range $\pi / 2<\phi<3 \pi / 2$ respectively). These sets of points are in correspondence of perigee altitudes between 600 and $800 \mathrm{~km}$ approximately, where aerodynamic drag and solar radiation pressure have comparable effect. As expected from the analysis in Section 3.1, no global solution of Eq. (16) can be found here; however partial equilibrium solutions exist

\footnotetext{
${ }^{4}$ For easy of representation in Fig. 2 we simplify the notation of the three equations of system Eq. (16) to $\|\Delta a\|=0$, $\|\Delta e\|=0$, and $\|\Delta \phi\|=0$.
} 


$$
\begin{aligned}
& \left\{\begin{array}{l}
\left\langle\frac{d a}{d t}\right\rangle_{\mathrm{SRP}+\mathrm{Drag}}=0 \\
\left\langle\frac{d \tilde{\omega}}{d t}\right\rangle_{\mathrm{SRP}+J_{2}}-n_{\mathrm{Sun}}=0
\end{array}\right. \\
& \left\{\begin{array}{l}
\left\langle\frac{d e}{d t}\right\rangle_{\mathrm{SRP}+\mathrm{Drag}}=0 \\
\left\langle\frac{d \tilde{\omega}}{d t}\right\rangle_{\mathrm{SRP}+J_{2}}-n_{\mathrm{Sun}}=0
\end{array}\right.
\end{aligned}
$$

in which the Sun-synchronous apse-line condition is satisfied and only one variation, either semi-major axis or eccentricity is zero. Note that the global the multi-start algorithm can be used to identify the solution set of Eq. (18), or Eq. (19) by simply setting in Eq. (17) $w_{e}=0$ or $w_{a}=0$, respectively. The ratio between $w_{a}$ and $w_{\omega}$, and $w_{e}$ and $w_{\phi}$ were chosen such that, for any solution found, the Sun-synchronous apse-line condition is always satisfied (i.e., $w_{\phi}=10 \cdot w_{a}$ or $w_{\phi}=10 \cdot w_{e}$, respectively).

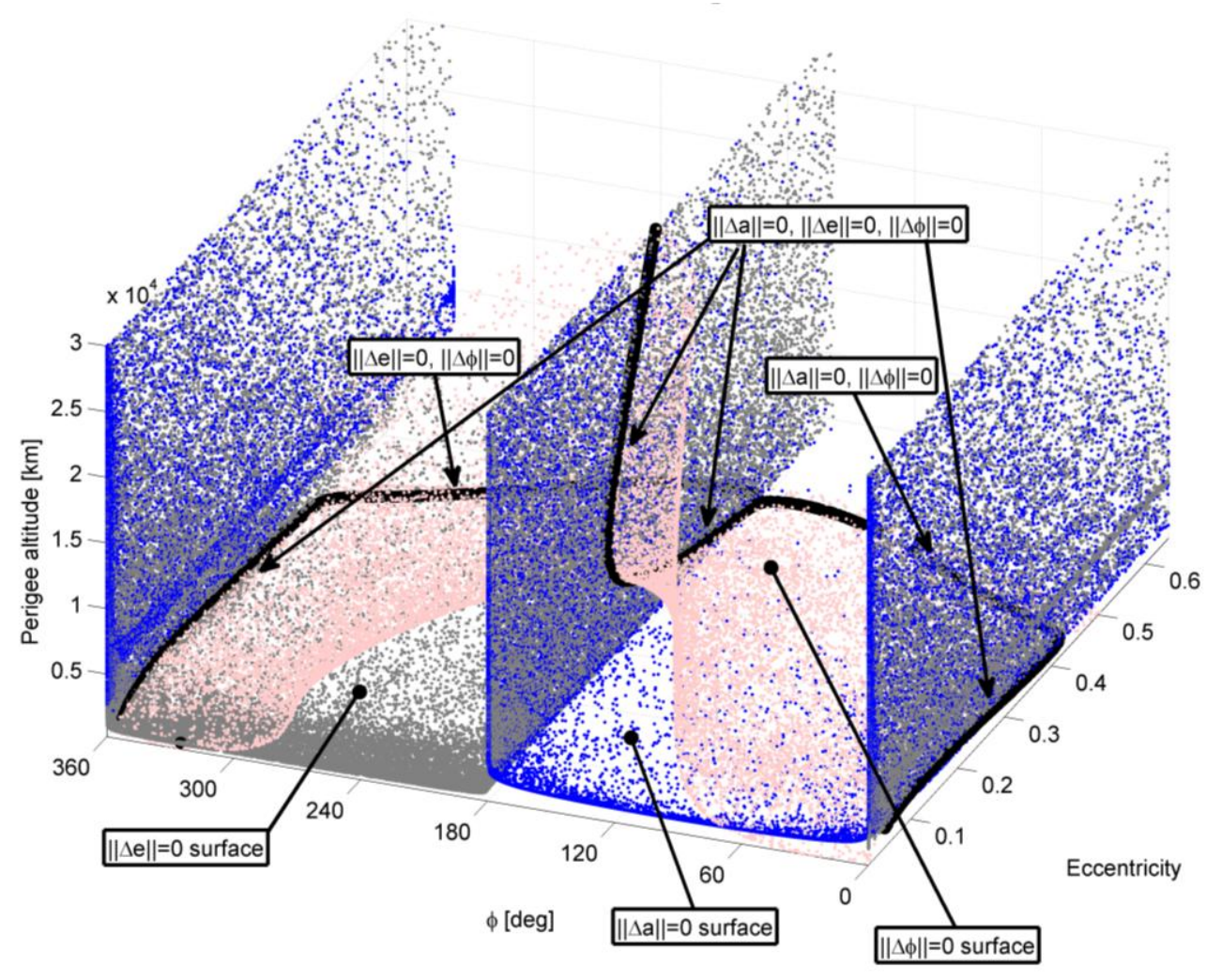




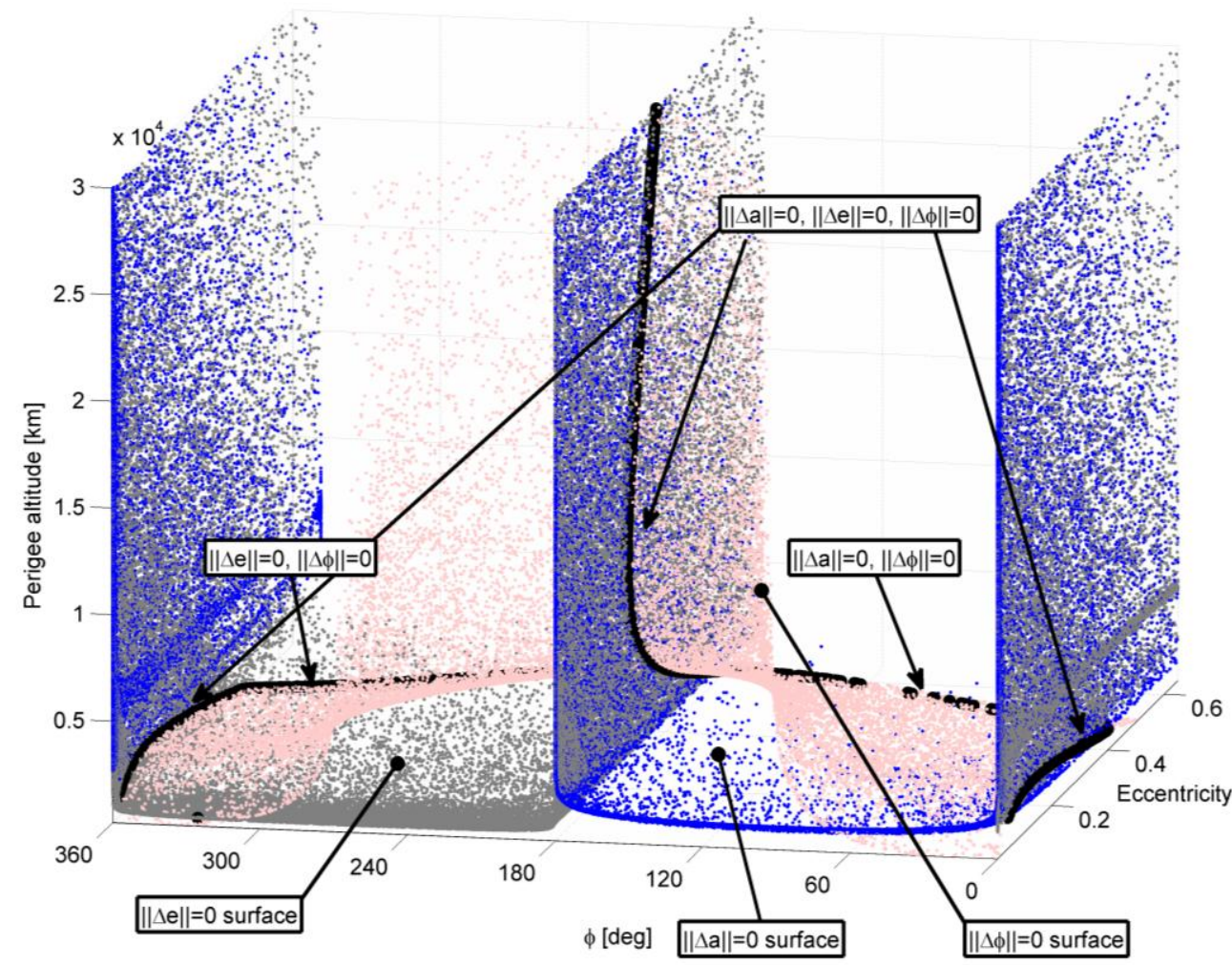

Fig. 2: Solution of system of Eqs. (16) for a SpaceChip whose characteristics are reported in Section 4.1. The intersection of the three surfaces represents equilibrium orbits.

In Fig. 3 the equilibrium solutions of Eq. (16), identified by the intersection of the three surfaces in Fig. 2, are reported (black lines) as a function of perigee altitude (Fig. 3a and b) and semi-major axis (Fig. 3c and d). The two families of anti-heliotropic and heliotropic orbits can be distinguished at $\phi=\pi$ and $\phi=0,2 \pi$ respectively. In the same graph we also compare the equivalent solutions found without considering eclipses (medium grey line) and with eclipses but neglecting $J_{2}$ (light grey line). The effect of eclipses at a fixed semi-major axis displaces the equilibrium points at lower eccentricities; the reason being that, when part of the orbit is in shadow, a lower variation of longitude of perigee is achieved through the influence of SRP (see the third of Eqs. (6)), hence a new equilibrium orbit is possible only at a lower eccentricity at which the change of $\langle d \tilde{\omega} / d t\rangle_{\mathrm{SRP}+J_{2}}$ is again able to balance $n_{\mathrm{Sun}}$. From

Fig. 3 it can also be seen how the effect of $J_{2}$ influences the equilibria; indeed, the solution considering a spherical gravity field (light grey line) drives away from the solution considering an oblate gravity field (black line) as the semi-major axis decreases. 

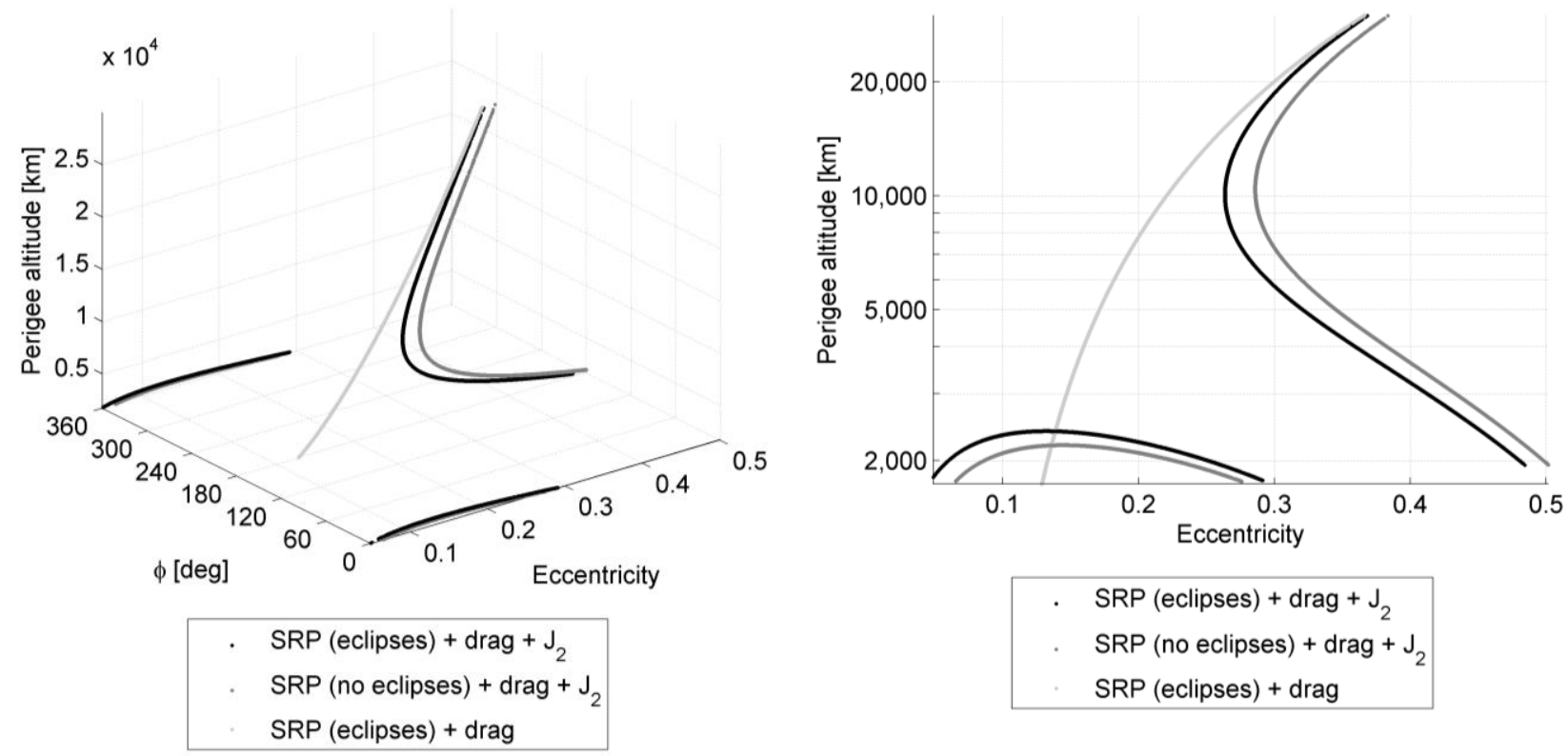

a)

b)
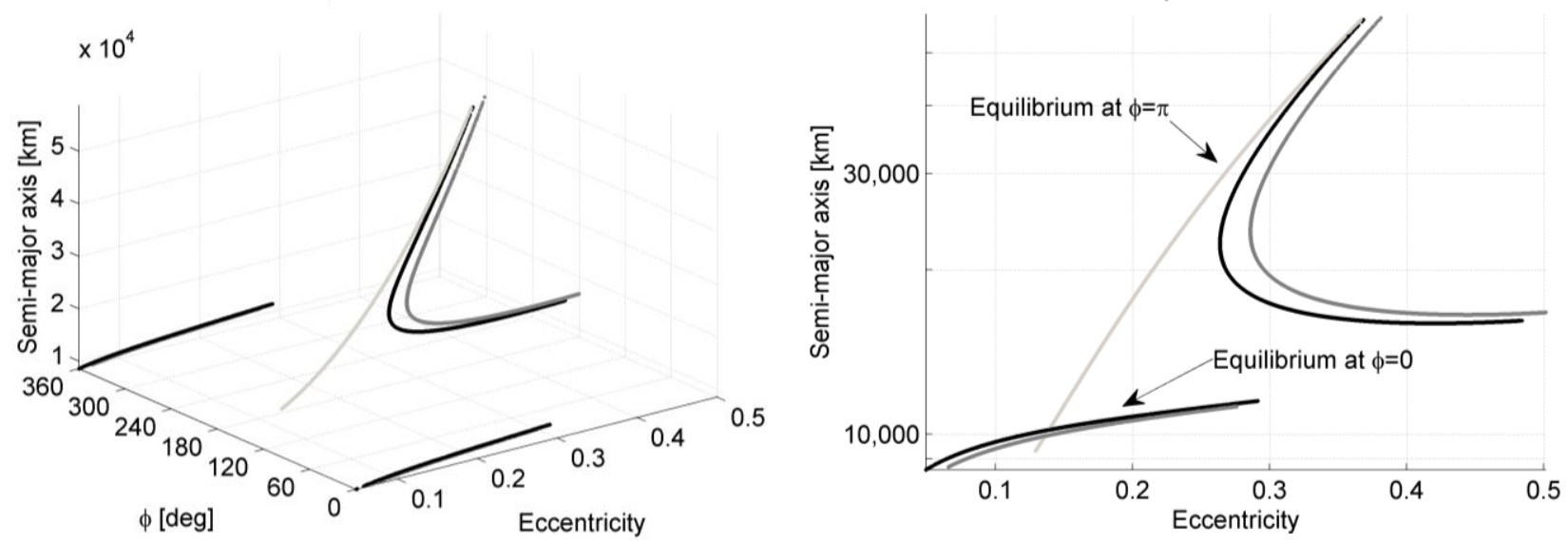

d)

Fig. 3: Equilibrium orbits (for the SpaceChip in Section 4.1) with SRP and drag (light grey line); SRP, drag and $J_{2}$ (black line); and SRP, drag and $J_{2}$ with no eclipses (medium grey line).

\subsection{Long-term orbit evolution}

As has been noted, solutions of Eq. (16) identify conditions for equilibrium orbits in which the orbital elements remain fixed under the effect of asymmetric solar radiation pressure, $J_{2}$, and atmospheric drag. Only some periodic variation in the elements will be experienced, but their average over a full orbit revolution vanishes.

Other initial conditions in the $\{e, \phi, a\}$ space will instead evolve under the influence of orbit perturbations. The long-term evolution of these conditions can be verified through numerical integration of Eqs. (14) and some insights can be drawn by observing the zero surfaces for $\langle d a / d t\rangle_{\mathrm{SRP}+\text { Drag }},\langle d e / d t\rangle_{\mathrm{SRP}+\text { Drag }}$, and $\langle d \phi / d t\rangle_{\mathrm{SRP}+J_{2}}$ (respectively blue, grey, and pink dotted surfaces in Fig. 2) and considering that the sign of the derivatives in the region of the element space marked off by those surfaces. $\langle d e / d t\rangle_{\mathrm{SRP}+\operatorname{Drag}}$ is negative for sets of orbital elements within the domain $0<\phi<\pi$, whereas in the region $\pi<\phi<2 \pi$ it is positive above the grey surface in Fig. 2 , and negative below. The opposite happens for semi-major axis: $\langle d a / d t\rangle_{\mathrm{SRP}+\text { Drag }}$ is negative for any set of $\{e, \phi, a\}$ in the domain $\pi<\phi<2 \pi$; within the range $0<\phi<\pi$, instead, it is positive above the blue surface in Fig. 2, and negative below. Finally, the 
pink surface Fig. 2 delimits the region of the element space of positive $\langle d \phi / d t\rangle_{\mathrm{SRP}+J_{2}}$ (region containing the line $e=0, \phi=\pi$ ), and negative $\langle d \phi / d t\rangle_{\mathrm{SRP}+J_{2}}$ (complementary region in the element space) [22].

As a consequence, choosing a certain initial condition in the element space, this will evolve in time with complicated effects arising from the interaction of the SRP and $J_{2}$ effect, as described for example in [18]. For altitudes below $2,000 \mathrm{~km}$, the motion will be also progressively decaying, due to the non-conservative influence of atmospheric drag. However, it is possible to define arcs of the orbit evolution where the trajectory is dominated by solar radiation pressure, such that the energy gain due to SRP balances the energy dissipation due to drag. Moreover, asymmetry in solar radiation pressure due to eclipses leads to modulation of the orbit energy and angular momentum [22]. This natural dynamics will be exploited in the next section, to distribute a swarm of SpaceChips in the upper layers of the Earth's atmosphere. In addition, the effect of atmospheric drag can be exploited to ensure the end-of-life decay of SpaceChips, thus preventing long-lived orbital debris.

\section{SpaceChip swarm missions}

The employment of MEMS devices for space applications offers significant potential, mainly due to the low cost of fabrication, and launch or deployment as piggy-back on a conventional spacecraft. Therefore, it is possible to identify new space mission concepts addressing goals that cannot be met with larger systems.

Firstly, such devices can be deployed in a swarm for real-time applications, to collect measurements over a spatially distributed domain. Their ideal application is for taking single measurements (temperature, detect the presence or absence or a particular pre-defined condition, etc.) rather than performing complex analysis on each single device [1]. Moreover, their disposability enables mission designs with high risk, since a lost device can be easily replaced, and the average behaviour of the swarm can be exploited, rather than the evolution of each satellite 5 $[4,8,9]$.

Swarms of SpaceChips can enable new missions, such as remote sensing for Earth observation, space weather, reflectometry and interferometry (oceanic properties such as surface height, significant wave height, wind speed and wind direction), spectrometry, and even more challenging missions for interplanetary and deep space exploration, in support of conventional spacecraft.

It was previously shown that Sun-synchronous apse-line precession can be artificially obtained with a SpaceChip corresponding to current nano-fabrication technologies [22]. This concept could be adapted to enhance the return of a GEOSAIL type mission [19, 20]. A swarm of SpaceChips can be used as distributed nodes of a network in the useful region of the element space, to obtain a spatial and temporal map of the geomagnetic tail, similar to the concept of the Kilo-Satellite constellation proposed by Petschek et al. [9].

In this paper we propose the employment of a SpaceChip swarm for mapping the upper stages of the atmosphere. In particular the ionosphere extends from approximately $80 \mathrm{~km}$ to $640 \mathrm{~km}$; in this region the gases which compose the Earth's atmosphere are highly rarefied, the atoms are ionised by solar radiation and the level of ionisation is influenced strongly by solar activity. An understanding of this region would be particularly interesting as conditions in the ionosphere influence the quality of transmission from communication satellites ${ }^{6}$ and can affect the accuracy of satellite navigation systems. At higher altitudes (up to approximately $1280 \mathrm{~km}$ ) the low-density region of the Earth's atmosphere extends to the exosphere, where atoms and molecules escape, due to scattering by solar wind particles and exchange of electrical charges.

\subsection{Spacecraft and perturbation model}

We consider a silicon microchip (density $2.3 \mathrm{~g} / \mathrm{cm}^{3}$ ) of $1 \times 1 \mathrm{~cm}^{2}$ and $0.025 \mathrm{~mm}$ thick, as designed by Atchison and Peck [1]. Table 1 reports the SpaceChip dimensions and the radius of a spherical particle with an equivalent area-to-mass ratio. The sphere shape is usually adopted for studies on interplanetary dust [11]. Since the SpaceChip density is assumed uniform, the characteristic length is represented by the chip's thickness $l_{2}$ :

$$
\begin{array}{ll}
A & =l_{1}^{2} \\
m & =l_{1}^{2} \cdot l_{2} \cdot \rho_{\text {silicon }}
\end{array} \quad \frac{A}{m}=\frac{1}{l_{2} \cdot \rho_{\text {silicon }}}
$$

\footnotetext{
${ }^{5}$ ChipSat workshop, 18 February 2010, Brown University, website: http://www.engin.brown.edu/chipsat/index.html [retrieved on 07 September 2010].

${ }^{6} \mathrm{http}: / /$ science.nasa.gov/missions/terriers/ [retrieved on 11 November 2011].
} 
where $A$ is the cross-section area. In this paper we consider that the average effective cross-section $A_{\odot}$ exposed to the Sun is always equal to the cross-sectional area of the spacecraft $A$. This implies that the spacecraft has a spherical shape or its attitude is kept fixed with respect to the Sun-Earth line. A passive Sun-pointing attitude control was proposed for millimetre-scale solar sails, based on faceted surfaces to stabilise a Sun-pointing plate [1]. Alternately, electro-chromic elements with variable reflectance can be layered at the sides of the chip to be exploited as a steering device, as demonstrated on the IKAROS mission [34]. Moreover, a reflectivity coefficient $c_{R}=1.8$ is assumed (note that for a black body $c_{R}=1$, for a flat mirror perpendicular to the light direction $c_{R}=2$ ).

For the model of atmospheric drag, a drag coefficient $c_{D}=2.1$ is chosen $\left(c_{D}=2.2\right.$ is usually used for a flat plate model, $c_{D}=2.0$ to 2.1 for spherical particles), and the cross-sectional area $A_{\text {Drag }}$ is considered constant and equal to the cross-sectional area of the spacecraft $A$. The reference values for the computation of the air density in Eq. (10) were taken as [27]:

$$
\begin{aligned}
& h_{0}=600 \mathrm{~km} \\
& \rho_{0}=1.454 \times 10^{-13} \mathrm{~kg} / \mathrm{m}^{3} \\
& H=71.835 \mathrm{~km}
\end{aligned}
$$

since the region where solar radiation pressure and atmospheric drag are known to have comparable effect is approximately around $400-800 \mathrm{~km}$. A more accurate value of the density would be obtained by selecting the reference values $h_{0}, \rho_{0}$ and $H$ closest to the range of orbit altitudes considered. Table 1 also contains the characteristic acceleration due to solar radiation pressure computed through Eq. (2).

Table 1: SpaceChip characteristics.

\begin{tabular}{lll}
\hline Parameter & Units & \multicolumn{1}{c}{ Value } \\
\hline Chip dimensions & {$[\mathrm{mm}]$} & $10 \times 10 \times 0.025$ \\
Sphere dimensions & {$[\mathrm{mm}]$} & 0.0187 \\
$\mathrm{~A} / m$ & {$\left[\mathrm{~m}^{2} / \mathrm{kg}\right]$} & 17.39 \\
$a_{\mathrm{SRP}}$ & {$\left[\mathrm{mm} / \mathrm{s}^{2}\right]$} & 0.1427 \\
\hline
\end{tabular}

The long-term evolution of the SpaceChips is predicted by integrating Eqs. (14) until the perigee altitude decreases below $h_{p} \leq 50 \mathrm{~km}$. This limit is set because below a certain perigee altitude the orbit rapidly decays and the mission is terminated. The numerical integration was performed through an adaptive step-size Runge-KuttaFehlberg integration scheme integrator with a six stage pair of approximation of the fourth and fifth order [35], with absolute and relative tolerance of $2.5 \times 10^{-14}$.

\subsection{Mission for mapping the upper layers of the atmosphere}

A larger, conventional spacecraft whose sizing is reported in Table 2 is injected into an Earth orbit lying in the equatorial plane, with initial eccentricity 0.12 and pericentre altitude of $600 \mathrm{~km}$. On this orbit, the spacecraft motion will be only influenced by the Earth's oblateness effect which causes a precession of the apse-line $\langle d \tilde{\omega} / d t\rangle_{s / c, J_{2}}$ of 4.8 degree per day, from Eq. (12c). It will therefore cover a range of $\phi$ from 0 to $2 \pi$ in approximately 95 days (corresponding to many orbits around the Earth):

$$
\Delta t_{s / c, 2 \pi-\phi}=\frac{2 \pi}{\langle d \tilde{\omega} / d t\rangle_{s / c, J_{2}}-n_{\mathrm{Sun}}}
$$

The carrier spacecraft motion is only marginally influenced by solar radiation pressure and atmospheric drag; therefore, its orbit will follow a quasi-straight line in the eccentricity- $\phi$ phase space. 
Table 2: Carrier spacecraft characteristics.

\begin{tabular}{cl}
\hline Parameter & Value \\
\hline$m$ & $100 \mathrm{~kg}$ \\
$A$ & $0.8 \mathrm{~m}^{2}$ \\
$c_{D}$ & 2.1 \\
$c_{R}$ & 1.2 \\
\hline
\end{tabular}

As this carrier spacecraft moves along its orbit, it will release a number of SpaceChips. Because of their high area-to-mass, the SpaceChip devices will not follow the carrier orbit (black line); rather their orbit will evolve under the effect of $J_{2}$, solar radiation pressure and drag, as shown in Fig. 4. The long-term evolution of the SpaceChip orbits after release is shown with the continuous blue line, corresponding to the initial conditions along the carrier spacecraft motion (represented by the green dots superimposed on the black line). As expected, their evolution depends on the orientation of the orbit perigee relative to the Sun. For $\phi<\pi$ and $h_{p}>600 \mathrm{~km}, \Delta a_{\mathrm{SRP}+\mathrm{Drag}}>0$ and $\Delta e_{\mathrm{SRP}+\mathrm{Drag}}<0$; as a consequence the orbit perigee rises reaching its local maximum at $\phi=\pi$ or $\phi=0$. Instead, when $\phi>\pi$ and $h_{p}>600 \mathrm{~km}, \Delta a_{\mathrm{SRP}+\mathrm{Drag}}<0$ and $\Delta e_{\mathrm{SRP}+\text { Drag }}>0$, hence the perigee altitude decreases. At an altitude below $600 \mathrm{~km}$, the influence of drag becomes predominant and causes the decay of the SpaceChip orbits as $\Delta a_{\mathrm{SRP}+\mathrm{Drag}}<0$ and $\Delta e_{\mathrm{SRP}+\mathrm{Drag}}<0$ (the blue circles indicates the final decay at an altitude below $50 \mathrm{~km}$ ). In Fig. 4 the orbit evolution under the effect of drag only is superimposed (green line), starting from the same initial conditions identified by the green dots. The orbit shrinks while the radius of the perigee tends to remain constant (see also Fig. $8)$.

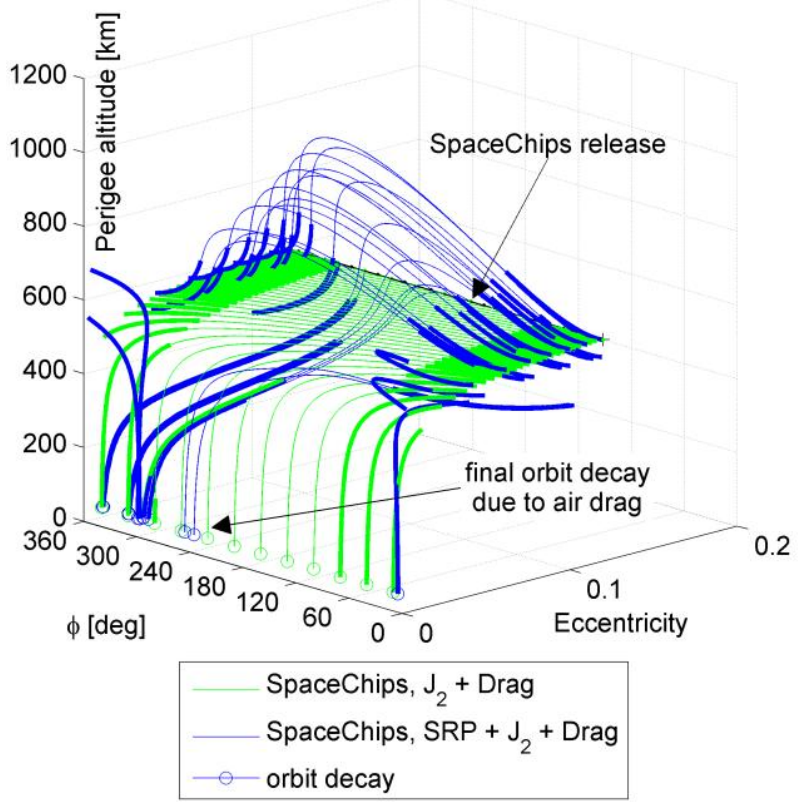

a)

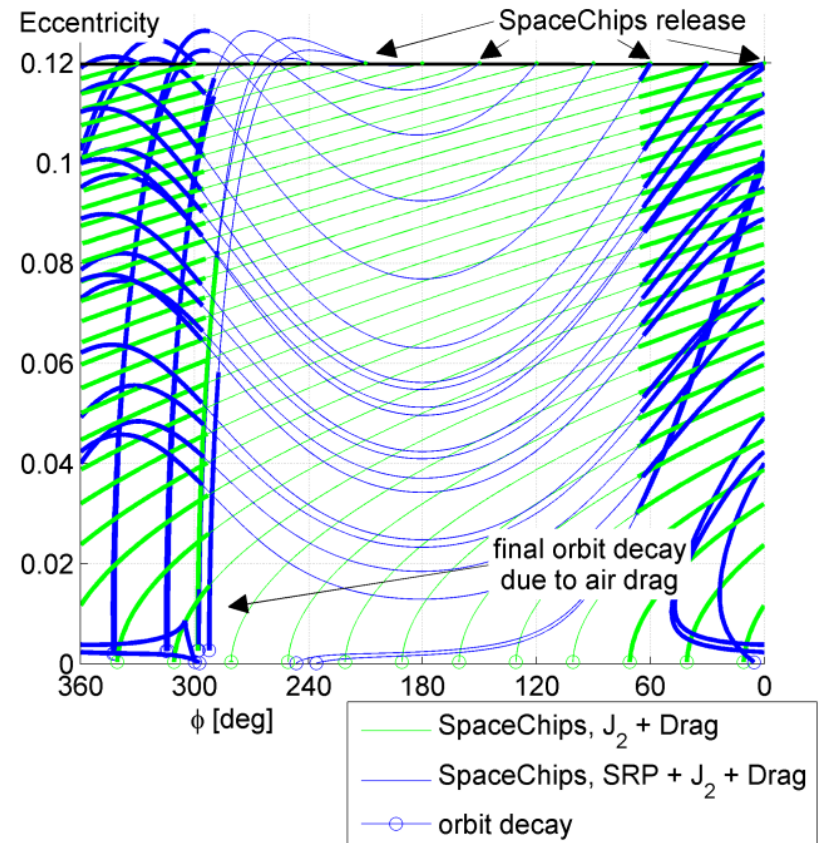

b)

Fig. 4: SpaceChip swarm evolution in the element space. The carrier spacecraft follows the black orbit; the long-term evolution of the released chips under the effect of SRP, $J_{2}$ and drag is represented by the blue line. The evolution under the effect of $J_{2}$ and drag is represented with the green line. The arc in which the perigee is in eclipse is highlighted with a bold line. a) View in eccentricity, $\phi$, and perigee altitude and b) view in eccentricity and $\phi$.

As example, Fig. 5 focuses on the phase space evolution of one particular release condition corresponding to $\phi_{s / c}=0$. As shown is Fig. 5a, while the carrier spacecraft continues its motion in the phase space on the black line, the SpaceChips after release evolve along the blue line. If no drag were experienced, the spacecraft would have 
covered indefinitely the red line ${ }^{7}$. In the case solar radiation pressure had no influence, instead, the evolution would follow the green line and the SpaceChips would rapidly decay. The long-term evolution of the SpaceChip swarm can be also represented in the $e-\phi$ polar reference frame, as shown in Fig. 5b. The dispenser orbit describes a circle over $\Delta t_{s / c, 2 \pi-\phi}$ (given by Eq. (20)). Through this representation it is easy to identify the angular displacement of the dispenser's orbit pericentre at the moment of the SpaceChip is released (i.e., $\phi_{s / c}=0$ ). The long-term evolution under SRP, $J_{2}$, and drag is indicated with the continuous blue line, whereas the evolution subject to drag and $J_{2}$ only is represented with the green line. The trajectory finally evolves towards the end-of-life decay at the origin of the graph.

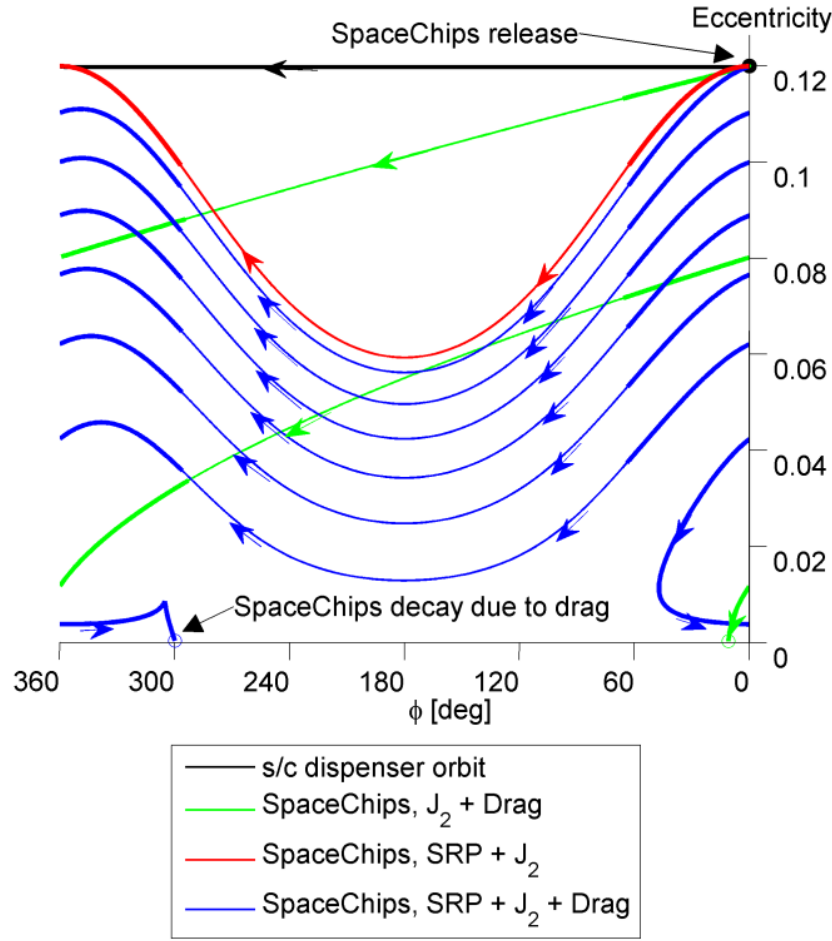

a)

Fig. 5: SpaceChip swarm evolution $\left(\phi_{s / c}=0 \mathrm{deg}\right)$ in the phase space. The carrier spacecraft follows the black line; the long-term evolution of the released chips under the effect of SRP, $J_{2}$ and drag is represented by the blue line. The evolution under the effect of drag and $J_{2}$ only is represented with the green line. The evolution under the effect of SRP and $J_{2}$ only is represented with the red line. The arc in which the perigee is in eclipse is highlighted with a bold line.

Fig. 6 shows the same evolution in an Earth centred reference frame, with its $x$-axis rotating with the Sun-Earth line. In fact each point on the evolution in the phase space (each point of the blue line in Fig. 5) represents a different subsequent orbit shape and orientation with respect to the Sun. The initial SpaceChip orbit is equal to the dispenser orbit (bold black orbit), then it evolves in time covering all the orbits represented from magenta to red colour, until the final decay phase, when the perigee quickly decreases.

\footnotetext{
${ }^{7}$ Note that, being the phase space periodic in $\phi$, once a line stops at $\phi=2 \pi$, this reappears at the same value of eccentricity in correspondence of $\phi=0$. The direction of motion is shown with the arrows.
} 


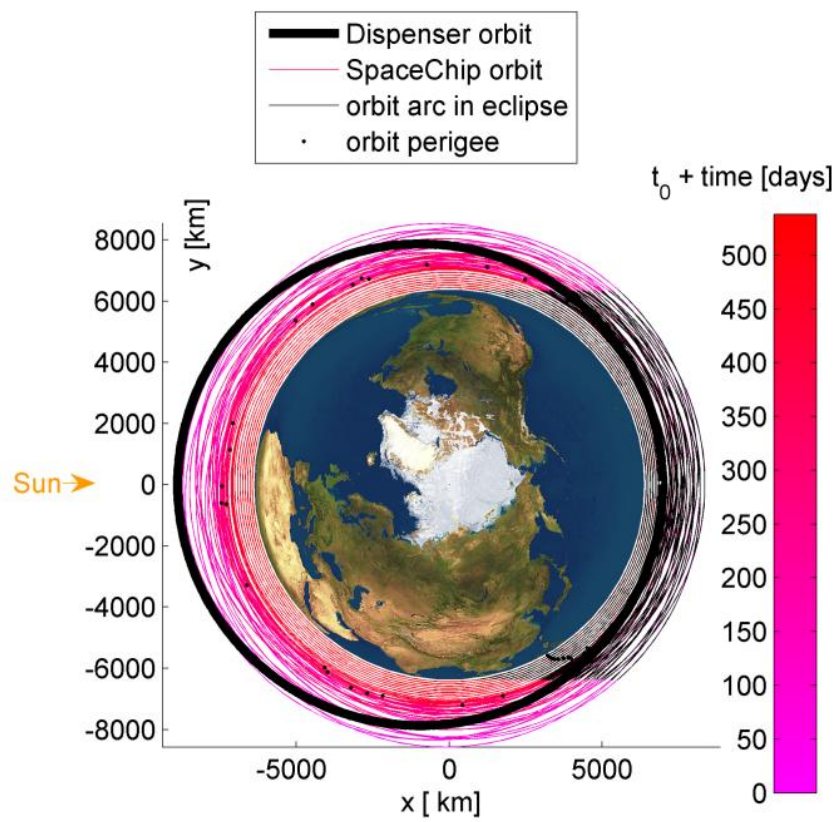

Fig. 6: SpaceChip orbit evolution ( $\phi_{s / c}=0 \mathrm{deg}$ ) with SRP, $\boldsymbol{J}_{2}$ and drag in an Earth centred reference frame. The arc of orbit in eclipse is highlighted with a black line. The colour bar indicates the elapsed time in days from release (magenta) to decay (red).

Fig. 7 represents the atmosphere coverage of the overall mission in terms of perigee and apogee altitudes (continuous blue line). The exploitation of SRP allows coverage of a more extended region of the atmosphere from the device release (black point) until the final decay (when the perigee altitude decreases below $50 \mathrm{~km}$ ). The swarm will explore an extended region of the ionosphere and the exosphere collecting distributed measurements.

The SpaceChips would evolve towards a fast decay if only drag were influencing their motion (green line). This illustrates the importance of the use of SRP to deliver a useful mission lifetime for such small devices with a high area-to-mass ratio. However, the effect of drag can also be exploited to obtain a fast decay of such 'smart dust' devices in the terminal phase of the mission, ensuring their end-of-life disposal and avoiding the creation of longlived space debris from swarm of devices.

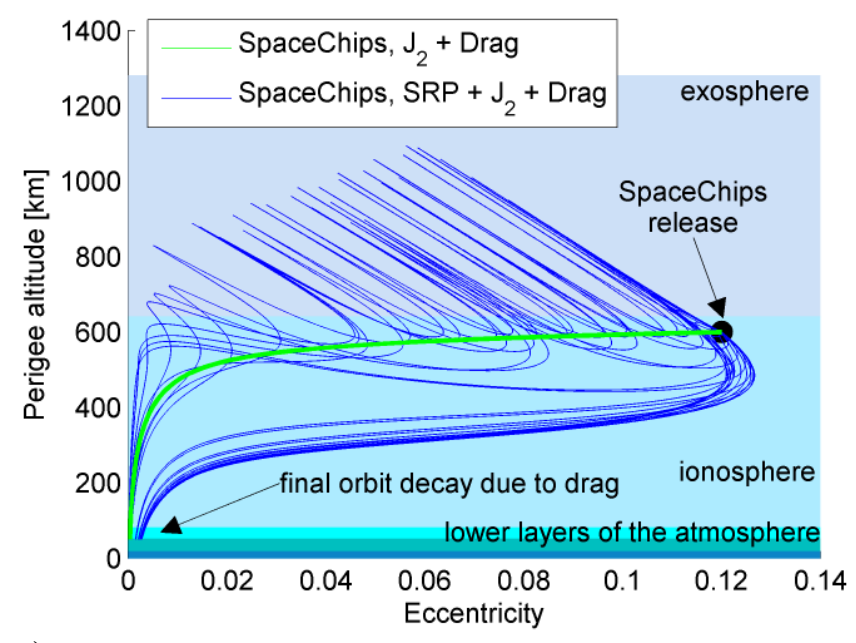

a)

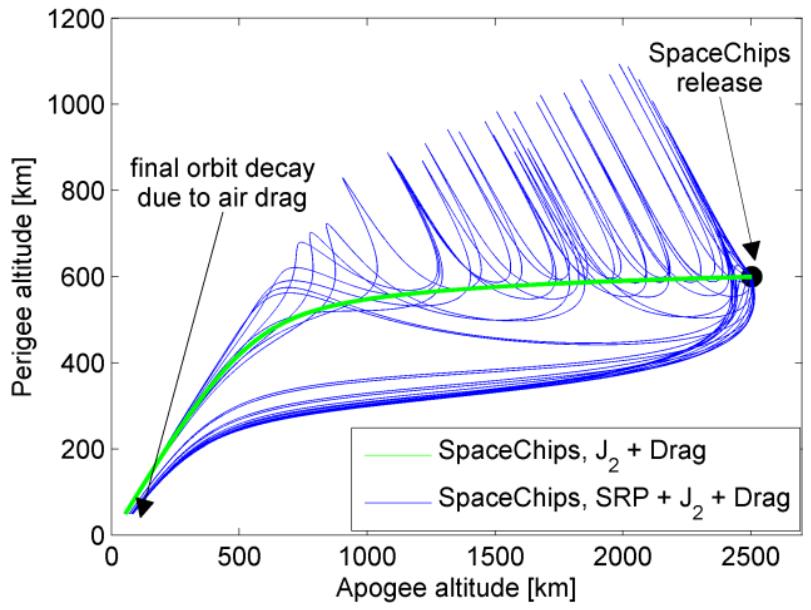

b) perigee and apogee altitudes. SpaceChips evolution under the effect of $J_{2}$, SRP and drag (blue line), and $J_{2}$ and drag only (green line). 
Fig. 8 shows the evolution of semi-major axis. By observing the blue line, corresponding to the arcs of the element space trajectory where the effect of SRP is dominant, the orbit energy is maintained almost constant (in fact, the energy slightly increases and then decreases during these arcs, due to eclipses). When the perigee altitude decreases below approximately $600 \mathrm{~km}$, due to the eccentricity oscillation at quasi-constant semi-major axis, the non-conservative effects of atmospheric drag dominate, hence the spacecraft experiences a rapid drop in semi major axis and will perform the next SRP-dominated loop at a lower value of the semi-major axis. In the case only $J_{2}$ and drag are present, the spacecraft experiences a continuous decrease in semi-major axis, until the orbit circularises and the orbit quickly decays (green line).

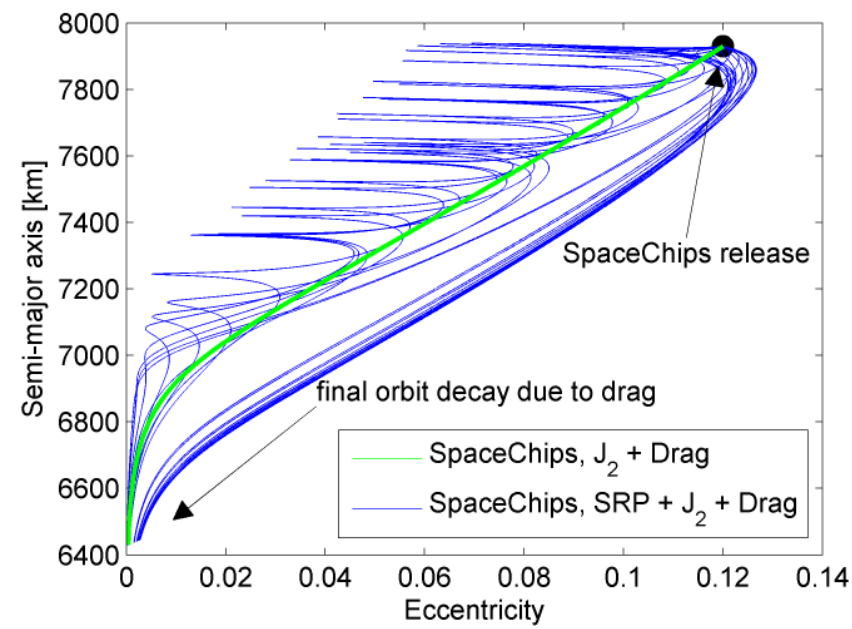

Fig. 8: Evolution of the semi-major axis as function of the eccentricity under the effect of $J_{2}$, SRP and drag (blue line), and $J_{2}$ and drag only (green line).

Depending on perigee orientation at release (i.e., angle $\phi$ ), the effect of SRP can significantly increase the orbit lifetime with respect to the drag-only case, as shown in Fig. 9a. If the SpaceChips are released when the carrier orbit apogee is oriented toward the Sun (around $\phi_{s / c}=0$ ), the coupled effect of $J_{2}$ and solar radiation pressure leads to a gain in orbit energy and a decrease of eccentricity to balance the dissipation due to air drag, as evidenced by the rotating trajectory in the orbital element space (Fig. 4). For this mission the maximum SpaceChip lifetime is up to 538 days (dotted blue line) with respect to a mission lifetime of 158 days without exploiting SRP (dotted green line). Moreover, the maximum perigee altitude reached during the mission can be increased up to $1095 \mathrm{~km}$ from the initial 600 km (see Fig. 9b).

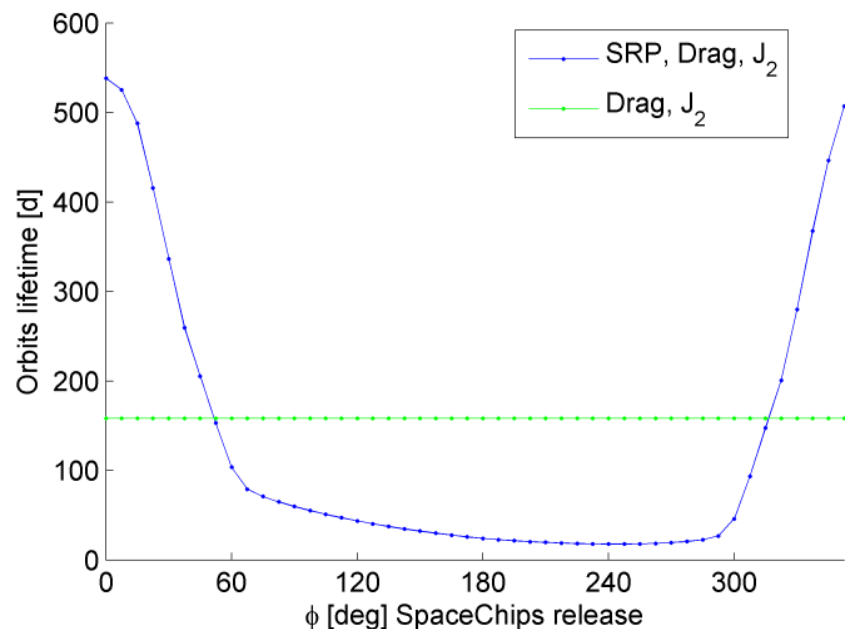

a)

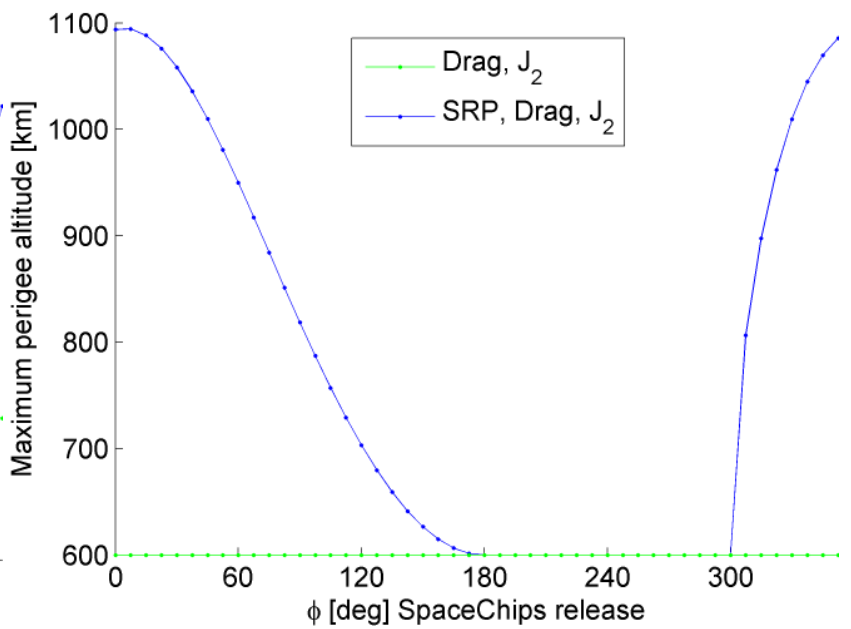

b)

Fig. 9: Orbit lifetime (a) and maximum perigee altitude (b) as function of the angular position of SpaceChip release under the effect of SRP, $J_{2}$, and drag (blue dotted line) and drag and $J_{2}$ only (green dotted line). 


\subsection{SpaceChip mission design}

The orbit of the carrier spacecraft can be used as design parameter to determine the lifetime of the SpaceChips after release and the maximum perigee they will reach in the following evolution. In the case that the eccentricity of the carrier spacecraft is also left as a mission design parameter, the orbit lifetime of the 'smart dust' devices and the maximum perigee altitudes reached are shown in Fig. 10. The perigee altitude at which the SpaceChips are released is chosen equal to $450 \mathrm{~km}$ (Fig. 10a) and $600 \mathrm{~km}$ (Fig. 10b), respectively, which corresponds to an increasing influence of atmospheric drag. Fixing the carrier perigee altitude, each combination of eccentricity and $\phi_{s / c}$ corresponds to a different initial condition for the SpaceChip release and its following evolution. The orbit lifetime is maximum if the initial condition is chosen close to the points which represent equilibrium orbits reported in Fig. 3. Therefore, the lifetime of SpaceChip orbits can be extended or reduced (with respect to the drag-only case) by exploiting SRP, and indeed selected depending on the release conditions. The maximum perigee altitude reached during the mission also depends on the release condition which determines the influence of solar radiation pressure over the subsequent orbit evolution. In particular, we observe a remarkable increase from the original perigee altitude of release when $0 \leq \phi_{s / c} \leq \pi$ for which the secular variation of $\langle d a / d t\rangle_{\mathrm{SRP}+\text { Drag }}$ is positive and the variation of $\langle d e / d t\rangle_{\mathrm{SRP}+\mathrm{Drag}}$ is negative.

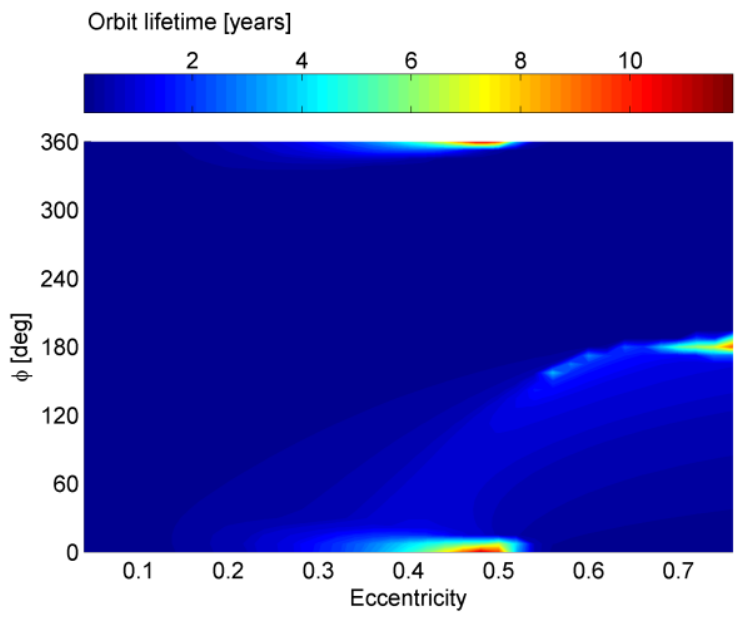

a) Carrier spacecraft at perigee altitude of $450 \mathrm{~km}$ Orbit lifetime [years]

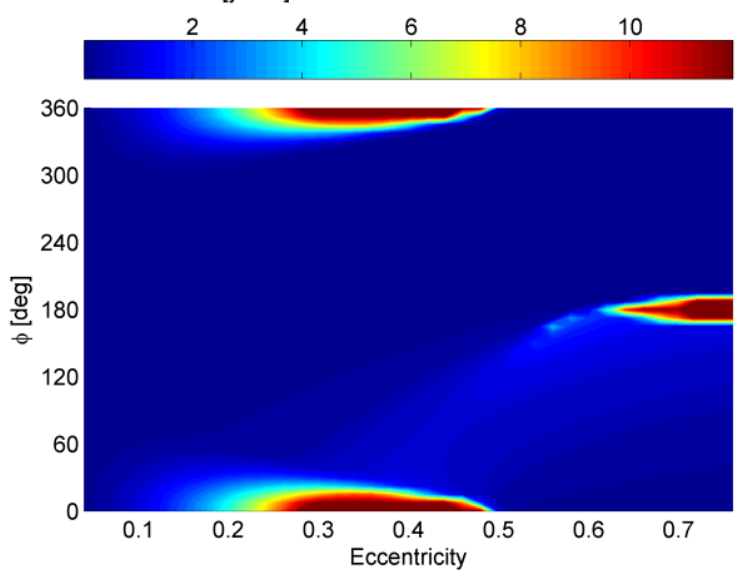

Max perigee altitude [km]

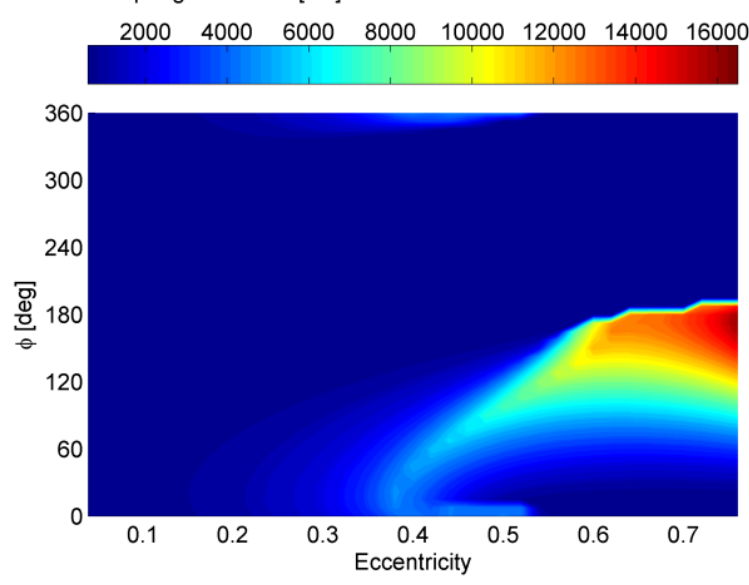

Max perigee altitude [km]

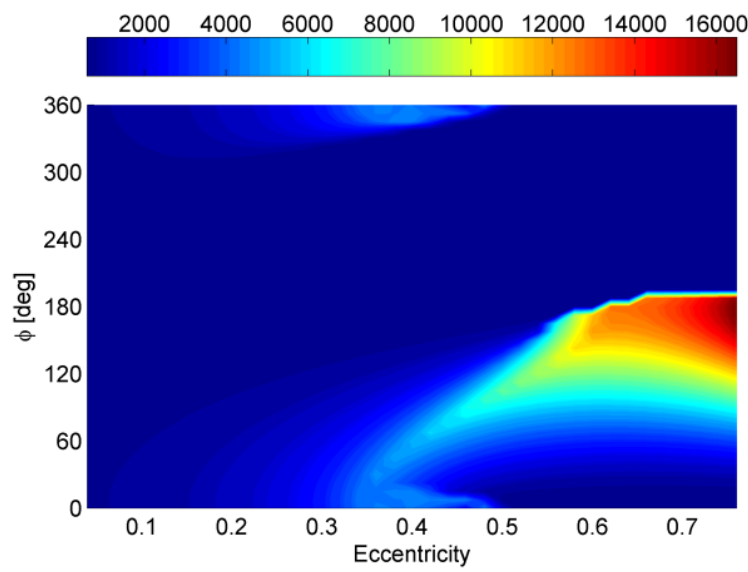

b) Carrier spacecraft at perigee altitude of $600 \mathrm{~km}$

Fig. 10: Orbit lifetime and maximum perigee altitude reached as function of the release conditions (angular position and eccentricity of SpaceChip release) under the effect of SRP, $J_{2}$, and drag. a) Perigee altitude of release of $450 \mathrm{~km}$, and b) $600 \mathrm{~km}$. 
We select, as an example, a more extended mission, in terms of perigee altitudes and total duration. The dispenser orbit has an initial eccentricity of 0.3 and perigee altitude of $600 \mathrm{~km}$. Also in this case the SpaceChip evolution is characterised by oscillations in eccentricity and perigee altitude as represented in Fig. 11 and Fig. 12. In particular, if the release condition is close to $\phi_{s / c}=0$ the SpaceChip will perform several rotational loops in the $e-\phi$ phase space. The orbit perigee precesses with respect to the Sun-Earth line (i.e., the orbit perigee moves faster than the Sun-Earth line), while the orbit stretches and contracts due to the oscillation both in eccentricity and semi-major axis (see the continuous blue line representing the evolution of the orbit pericentre in Fig. 12). Due to the effect of drag, a constant decaying motion is superimposed on the rotational motion. This is clearly visible in Fig. 12 which represents the evolution of the perigee altitude for all the release conditions. While in the case of drag and $J_{2}$ the perigee decays over a short time span (see green lines), for some release conditions under the effect of SRP, drag and $J_{2}$ the perigee oscillations cover a long time span as shown with the blue lines in Fig. 12 (i.e., the lifetime of the orbit evolution is extended). For those trajectories, the effect of drag is almost negligible over the major part of the rotational loop (i.e., oscillation of the perigee altitude between its local minimum and the following local maximum) and becomes predominant when the perigee reaches its local minimum; in this region the spacecraft experiences a rapid drop in orbit energy, therefore the following rotational loop will reach a lower value of the maximum orbit perigee altitude. The perigee oscillation due to SRP is damped by the presence of drag.

When the SpaceChip orbit evolution has more than one rotational loop in the element space, the orbit lifetime is consistently higher. This can be seen in Fig. 13a, which represents the orbit lifetime (from orbit injection up to decay below a perigee altitude of $50 \mathrm{~km}$ ) as a function of the angular displacement of release from the carrier spacecraft. When $\phi_{\mathrm{s} / \mathrm{c}}$ is close to zero the orbit lifetime reaches the maximum time limit of the integration set to 12 years.

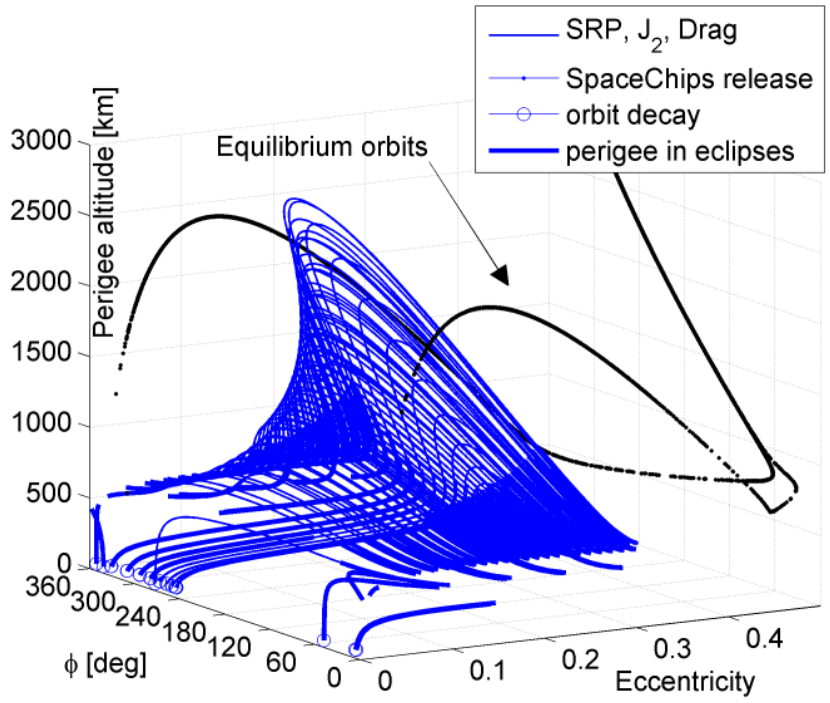

Fig. 11: SpaceChip swarm evolution in the element space. The long-term evolution of the released chips under the effect of SRP, $J_{2}$ and drag is represented by the blue line and the arcs in which the perigee is in eclipse is highlighted with a bold line. The black dots represent the condition for equilibrium and partial equilibrium orbits. 


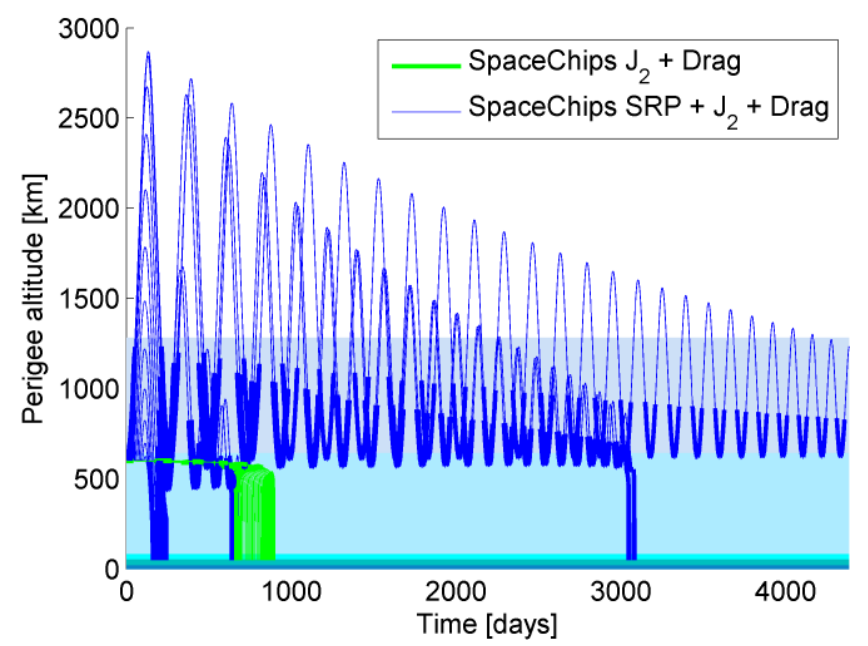

Fig. 12: Evolution of the perigee altitude under the effect of $J_{2}$, SRP and drag (blue line), and $J_{2}$ and drag only (green line).
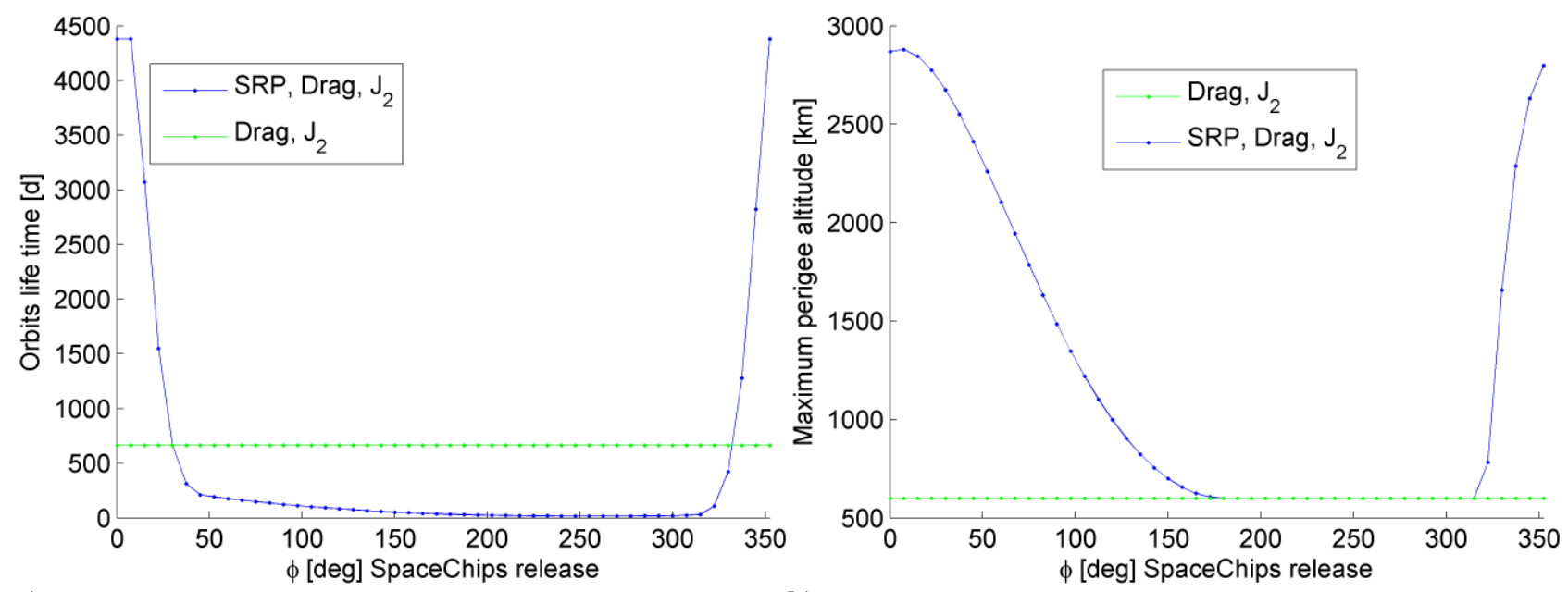

b)

Fig. 13 Orbit lifetime (a) and maximum perigee altitude (b) as function of the angular position of SpaceChip release under the effect of SRP, $J_{2}$, and drag (blue dotted line) and drag and $J_{2}$ only (green dotted line).

The range of maximum altitudes covered in the atmosphere is higher than the altitudes of the mission in Section 4.2, as can be seen in Fig. 14. The evolution of the SpaceChip swarm is represented with the continuous blue line and the initial orbit of release is shown with the black dot. 


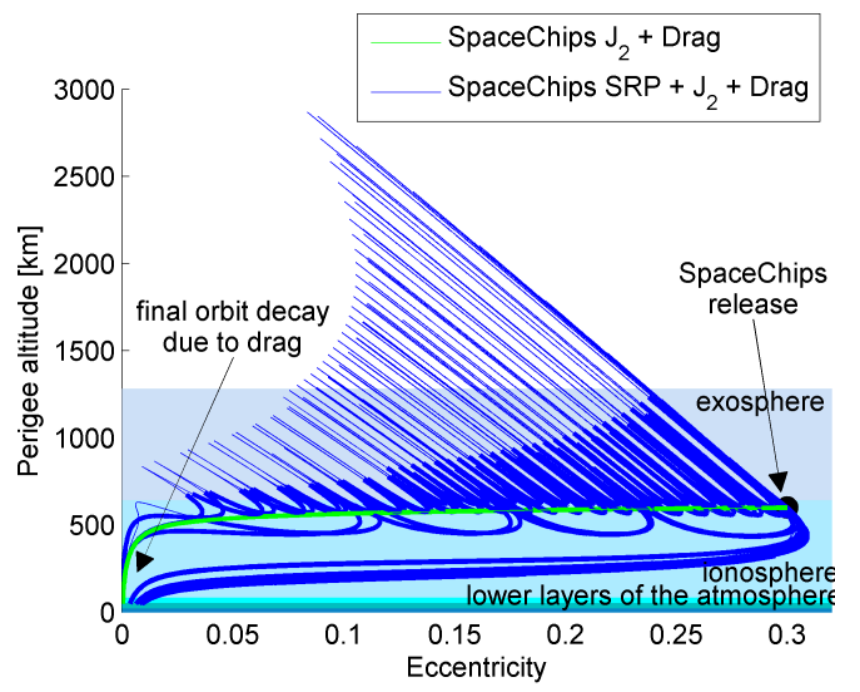

Fig. 14: Perigee altitude covered over the mission as function of the eccentricity. SpaceChip evolution under the effect of $J_{2}$, SRP and drag (blue line); and $J_{2}$ and drag only (green line).

\section{Discussion}

It may be inferred that a swarm of SpaceChips could represent a threat for conventional spacecraft orbiting at those altitudes due to the collision hazard [36]. The aim of this paper is to show that the effect of natural perturbations can be exploited to design operational orbits for very small devices and to select initial conditions for their deployment such that distributed measurements can be performed in a defined region of the element space. The orbit lifetime can be extended by exploiting SRP, but remains anyway limited by the effect of atmospheric drag (i.e., less than 25 years). Indeed, the end-of-life phase of the swarm mission can be designed, through passive re-entry in the lower regions of the atmosphere. Moreover, a more active way to control SpaceChip orbit evolution can be implemented, by changing the reflectivity coefficient of an electrochromic coating on the spacecraft and hence modulating the effect of solar radiation pressure on its orbit [37]. Through this technique the mission concept proposed in this paper could be extended by including active orbit control over the long-term evolution of the swarm. In this way the SpaceChips could be stabilised in a bounded region of the element space and the orbit lifetime of the swarm can be controlled [38].

Moreover, the concept presented in this paper could be adopted for the exploration of other inner planets, where orbit debris issue is not an issue and the effect of SRP exploited to disperse devices in the planetary atmosphere, offering the basis for planetary high altitude atmospheric studies. As pointed out by Atchison et al., a SpaceChip may be capable of decelerating and maintain a survivable temperature during re-entry into an atmosphere, with on-board electronics operating throughout the manoeuvre [39]. This possibility could enable distributed exploration of planetary surfaces, enhancing the data collection of a conventional spacecraft that can act as data rely [40]. All these concepts are also supported by low production cost and reduced masses of SpaceChips that can be easily stored as an additional payload. A trade-off could be also made with the alternative option of employing a swarm of small satellites (e.g., CubeSats) equipped with area-to-mass increasing devices (i.e., deployable reflecting balloon or small sail). This option would offer more complex sensing and observation capabilities at higher production and launch/deployment costs.

Finally, some considerations can be provided on the global coverage offered by a distributed sensing mission. Imagining that the SpaceChips are continuously released from the conventional spacecraft along its orbit, after few orbits of the carrier spacecraft a ring of 'smart dust' devices is assembled. In time the ring will evolve following one of the evolutions represented for example by the blue lines in Fig. 4 or Fig. 11, depending on the position of the carrier perigee at the moment of release (angle $\phi_{s / c}$ ). On the orbit, the SpaceChips will be compressed in number density close to apocentre, corresponding to the minimum of orbital angular velocity around the elliptical ring, and rarefied at pericentre corresponding to the maximum of orbital angular velocity. Hence, the elliptical ring of spacecraft will form wave-like patterns which circulate around the elliptical ring, with peaks in density at the apogee 
[41]. Considering, for example, the mission in Section 4.2, the carrier spacecraft takes 95 days $^{8}$ to cover a vertical line in the eccentricity $-\phi$ phase space (i.e. its orbit apse-line will cover all the possible orientations with respect to the Sun-Earth line), while the decay time of the SpaceChip orbit is between 538 days (for release conditions close to $\phi_{s / c}=0$ ) and less than 100 days (for release condition close to $\phi_{s / c}=\pi$ ). Hence, while the SpaceChip orbits evolve in the element space towards decay, the ring can be repopulated as long as the carrier spacecraft continues releasing devices along its orbit. Future studies will be aimed at addressing the simultaneous global coverage that can be achieved.

\section{Conclusions}

This paper analyses the orbital dynamics of high area-to-mass spacecraft under the influence of solar radiation pressure and atmospheric drag in an oblate gravity field. Initial conditions for long-lived orbits were determined by exploiting energy gain from asymmetric solar radiation pressure to offset the effect of energy dissipation due to atmospheric drag and the influence of the oblateness of the Earth is analysed.

The natural dynamics were exploited to design a mission for the investigation of the upper layers of the Earth's atmosphere which deploys a swarm of SpaceChips from a conventional carrier spacecraft. Due to the high area-tomass ratio of these 'smart dust' devices, the coupled effect of atmospheric drag and solar radiation pressure, with asymmetry due to eclipses, in an oblate gravity field, can be used to extend and select the orbit lifetime of such devices and design the disposal of the devices at the end of mission. The swarm covers perigee altitudes between 300 and $3000 \mathrm{~km}$, allowing distributed measurements of the conditions in the ionosphere and exosphere. The orbit lifetime can be extended by exploiting solar radiation pressure, but remains anyway limited by the effect of atmospheric drag (i.e., less than 25 years). Indeed, the end-of-life of behaviour of the swarm can be designed, through passive re-entry in the lower regions of the atmosphere.

\section{Acknowledgements}

This work was funded by the European Research Council, as part of project VISIONSPACE (227571).

\section{References}

1. Atchison, J. A. and Peck, M. A., "A Passive, Sun-Pointing, Millimeter-Scale Solar Sail,” Acta Astronautica, Vol. 67, No. 1-2, 2010, pp. 108-121. doi: 10.1016/j.actaastro.2009.12.008

2. Warneke, B. A. and Pister, K. S. J., "MEMS for Distributed Wireless Sensor Networks," Proceedings of the $9^{\text {th }}$ International Conference on Electronics, Circuits and Systems, Vol. 1, 2002, pp. 291-294.

3. Warneke, B., Last, M., Liebowitz, B. and Pister, K. S. J., "Smart Dust: Communicating with a CubicMillimeter Computer," Computer, Vol. 34, No. 1, 2001, pp. 44-51.

4. Vladimirova, T., Xiaofeng, W. and Bridges, C. P., "Development of a Satellite Sensor Network for Future Space Missions," Proceedings of the Aerospace Conference, 2008 IEEE, 2008, pp. 1-10.

5. Barnhart, D. J., Vladimirova, T. and Sweeting, M. N., "Very-Small-Satellite Design for Distributed Space Missions," Journal of Spacecraft and Rockets, Vol. 44, No. 6, 2007, pp. 1294-1306. doi: 10.2514/1.28678

6. Miller, L. M., "MEMS for Space Applications," Symposium on Design, Test and Microfabrication of MEMS/MOEMS - Review Paper, Paris, France, 1999, pp. 1-11.

7. Janson, S. W., "Silicon Satellites: Picosats Nanonats, and Microsats," Proceedings of the International Conference on Integrated Micro/Nanotechnology for Space Applications, The Aerospace Press, Houston, Texas, 1995.

8. Shapiro, I. I., Jones, H. M. and Perkins, C. W., "Orbital Properties of the West Ford Dipole Belt," Proceedings of the IEEE, Vol. 52, No. 5, 1964, pp. 469-518.

9. Petschek, H. E., Rayburn, C., Sheldon, R., Vickers, J., Bellino, M., Bevis, G. and Spence, H. E., Science Closure and Enabling Technologies for Constellation Class Missions, Library of Congress Cataloging-inPublication Data, University of California, Berkeley, 1998, pp. 51-57.

10. Atchison, J. A. and Peck, M. A., "Length Scaling in Spacecraft Dynamics," Journal of Guidance, Control, and Dynamics, Vol. 34, No. 1, 2011, pp. 231-246. doi: doi: 10.2514/1.49383

\footnotetext{
${ }^{8}$ It would instead take a year in a spherically symmetric gravity field.
} 
11. Hamilton, D. P. and Krivov, A. V., "Circumplanetary Dust Dynamics: Effects of Solar Gravity, Radiation Pressure, Planetary Oblateness, and Electromagnetism,” Icarus, Vol. 123, No. 2, 1996, pp. 503-523. doi: 10.1006/icar.1996.0175

12. Horányi, M. and Burns, J. A., "Charged Dust Dynamics: Orbital Resonance Due to Planetary Shadows," J. Geophys. Res., Vol. 96, No. A11, 1991, pp. 19283-19289. doi: 10.1029/91ja01982

13. Krivov, A. V., Sokolov, L. L. and Dikarev, V. V., "Dynamics of Mars-Orbiting Dust: Effects of Light Pressure and Planetary Oblateness," Celestial Mechanics and Dynamical Astronomy, Vol. 63, No. 3, 1995, pp. 313-339. doi: 10.1007/bf00692293

14. Anselmo, L. and Pardini, C., "Long-Term Dynamical Evolution of High Area-to-Mass Ratio Debris Released into High Earth Orbits," Acta Astronautica, Vol. 67, No. 1-2, 2010, pp. 204-216. doi: 10.1016/j.actaastro.2009.10.017

15. Schildknecht, T., Musci, R. and Flohrer, T., "Properties of the High Area-to-Mass Ratio Space Debris Population at High Altitudes," Advances in Space Research, Vol. 41, No. 7, 2008, pp. 1039-1045. doi: 10.1016/j.asr.2007.01.045

16. Shapiro, I. I. and Jones, H. M., "Perturbations of the Orbit of the Echo Balloon," Science, Vol. 132, No. 3438, 1960, pp. 1484-1486. doi: 10.1126/science.132.3438.1484

17. Musen, P., Bryant, R. and Bailie, A., "Perturbations in Perigee Height of Vanguard I," Science, Vol. 131, No. 3404, 1960, pp. 935-936. doi: 10.1126/science.131.3404.935

18. Krivov, A. V. and Getino, J., “Orbital Evolution of High-Altitude Balloon Satellites,” Astron. Astrophys., No. 318, 1997, p. 7.

19. McInnes, C. R., Macdonald, M., Angelopolous, V. and Alexander, D., "Geosail: Exploring the Geomagnetic Tail Using a Small Solar Sail," Journal of Spacecraft and Rockets, Vol. 38, No. 4, 2001, pp. 622-629.

20. Oyama, T., Yamakawa, H. and Omura, Y., “Orbital Dynamics of Solar Sails for Geomagnetic Tail Exploration," Journal of Guidance, Control and Dynamics, Vol. 45, No. 2, 2008, pp. 316-323. doi: 10.2514/1.31274

21. Mengali, G. and Quarta, A. A., "Near-Optimal Solar-Sail Orbit-Raising from Low Earth Orbit," Journal of Spacecraft and Rockets, Vol. 42, No. 5, 2005, pp. 954-958.

22. Colombo, C. and McInnes, C., "Orbital Dynamics of 'Smart Dust' Devices with Solar Radiation Pressure and Drag," Journal of Guidance, Control, and Dynamics, Vol. 34, No. 6, 2011, pp. 1613-1631. doi: $10.2514 / 1.52140$

23. Colombo, C., Lücking, C. and McInnes, C., "Orbital Dynamics of High Area-to-Mass Ratio Spacecraft under the Influence of $J_{2}$ and Solar Radiation Pressure," $62^{\text {nd }}$ International Astronautical Congress, Cape Town, 2011, IAC-11.C1.4.8.

24. Hamilton, D. P., “The Asymmetric Time-Variable Rings of Mars,” Icarus, Vol. 119, No. 1, 1996, pp. 153172. doi: 10.1006/icar.1996.0008

25. Fortescue, P., Stark, J. and Swinerd, G. (eds.), Spacecraft System Engineering, Third Edition ed. Wiley, Chichester, 2003.

26. King-Hele, D., Theory of Satellite Orbits in an Atmosphere, Butterworths, London, 1964.

27. Vallado, D. A., Fundamentals of Astrodynamics and Applications, Third Edition, Space Technology Library, New York, 2007.

28. Blitzer, L., "Handbook of Orbital Perturbations," University of Arizona, 1970.

29. Abramowitz, M. and Stegun, I. A., Handbook of Mathematical Functions, Applied Math. Series \#55, Dover Publications, 1965, Ch. 9.

30. Draim, J. E., Cefola, P. and Castrel, D., "Elliptical Orbit Constellations-a New Paradigm for Higher Efficiency in Space Systems?," Proceedings of the Aerospace Conference Proceedings, 2000 IEEE, Vol. 7 , 2000, pp. 27-35.

31. Colombo, C. and McInnes, C., "Orbital Dynamics of Earth-Orbiting 'Smart Dust' Spacecraft under the Effects of Solar Radiation Pressure and Aerodynamic Drag," AIAA/AAS Astrodynamics Specialist Conference 2010, Toronto, Canada, 2010, AIAA 2010-7656.

32. Coleman, T. F. and Li, Y., "On the Convergence of Interior-Reflective Newton Methods for Nonlinear Minimization Subject to Bounds," Mathematical Programming, Vol. 67, No. 1, 1994, pp. 189-224.

33. Coleman, T. F. and Li, Y., "An Interior Trust Region Approach for Nonlinear Minimization Subject to Bounds," SIAM Journal on Optimization, Vol. 6, No. 2, 1996, pp. 418-445. 
34. Kawaguchi, J. i., Mimasu, Y., Mori, O., Funase, R., Yamamoto, T. and Tsuda, Y., "IKAROS - Ready for Lift-Off as the World's First Solar Sail Demonstration in Interplanetary Space," Proceedings of the $60^{\text {th }}$ International Astronautical Congress, IAC 2009, Daejeon, Korea, 2009, IAC-09-D1.1.3.

35. Dormand, J. R. and Prince, P. J., "A Family of Embedded Runge-Kutta Formulae," Journal of computational and applied mathematics, Vol. 6, No. 1, 1980, pp. 19-26.

36. Rossi, A. and Valsecchi, G., "Collision Risk against Space Debris in Earth Orbits," Celestial Mechanics and Dynamical Astronomy, Vol. 95, No. 1, 2006, pp. 345-356. doi: 10.1007/s10569-006-9028-7

37. Lücking, C., Colombo, C. and McInnes, C., "Electrochromic Orbit Control for Smart-Dust Devices," Journal of Guidance, Control and Dynamics, accepted for publication, 2012.

38. Colombo, C., Lücking, C. M. and McInnes, C. R., "Orbit Evolution, Maintenance and Disposal of Spacechip Swarms," $6^{\text {th }}$ International Workshop on Satellite Constellation and Formation Flying (IWSCFF 2010), Taipei, Taiwan, 2010, IWSCFF-Paper-2010-2-2.

39. Atchison, J. A., Manchester, Z. R. and Peck, M. A., "Microscale Atmospheric Re-Entry Sensors," International Planetary Probe Workshop 2010 (IPPW-7), Barcelona,Spain, 2010.

40. Graziano, M., Cadenas, R., Medina, A., Puiatti, A., Mura, M., Puccinelli, D., Barrientos, A., Rossi, C., Sanz, D. and Dufour, J.-F., "Distributed Instruments in Preparation to Manned Missions to Mars and Moon," 61st International Astronautical Congress, Prague, 2010, IAC-10-D3.2.9.

41. McInnes, C. R. and Colombo, C., "Wave-Like Patterns in an Elliptical Satellite Ring," submitted to Journal of Guidance, Control, and Dynamics, 2011.

\section{Vitae}

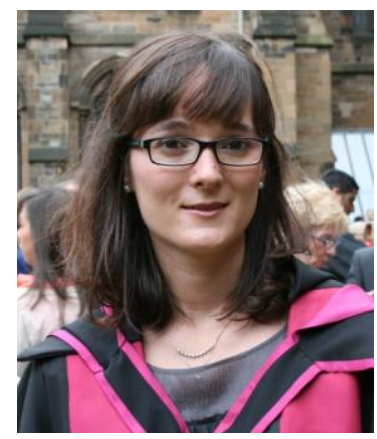

Camilla Colombo is Research Fellow at the Advanced Space Concepts Laboratory at the University of Strathclyde (UK). Her research focuses on the orbital dynamics of high area-to-mass spacecraft, space trajectory design and optimisation, and asteroid deflection. Previous to her current position she was appointed as research associate at the Department of Aerospace Engineering of the University of Glasgow (UK), where she completed her Ph.D. on "Optimal Trajectory Design for Interception and Deflection of Near Earth Objects" and was awarded the "William Wilson Scott Award Scholarship" (2009). She received her M.Sc. in Aerospace Engineering at Politecnico di Milano (Italy) with a thesis on an optimisation method for low-thrust trajectories based on Differential Dynamic Programming. 


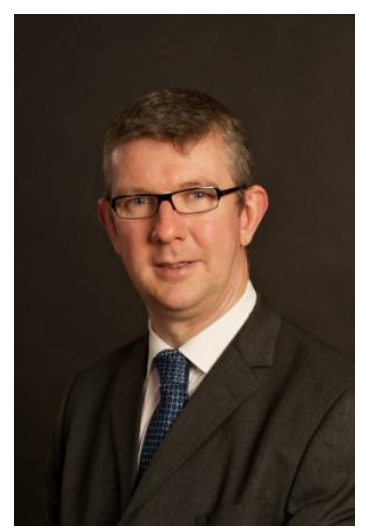

Colin McInnes is Director of the Advanced Space Concepts Laboratory at the University of Strathclyde. His work spans highly non-Keplerian orbits, orbital dynamics and mission applications for solar sails, spacecraft control using artificial potential field methods and is reported in over 100 journal papers. Recent work is exploring new approaches to spacecraft orbital dynamics at extremes of spacecraft length-scale to underpin future space-derived products and services. McInnes has been the recipient of awards including the Royal Aeronautical Society Pardoe Space Award (2000), and a Leonov medal by the International Association of Space Explorers (2007). The Advanced Space Concepts Laboratory was awarded the 2011 Sir Arthur C Clarke prize for Achievement in Space Research, 